\title{
Control and Synchronization of Chaotic and Hyperchaotic Lorenz Systems via Extended Backstepping Techniques
}

\author{
O. S. Onma, ${ }^{1}$ O. I. Olusola, ${ }^{1}$ and A. N. jjah $^{2}$ \\ ${ }^{1}$ Nonlinear Dynamics Research Group, Department of Physics, Federal University of Agriculture, PMB 2240, Abeokuta, Nigeria \\ ${ }^{2}$ Department of Physics, University of Lagos, Akoka, Lagos, Nigeria
}

Correspondence should be addressed to O. I. Olusola; olasunkanmi2000@gmail.com

Received 10 September 2013; Revised 15 November 2013; Accepted 17 November 2013; Published 6 May 2014

Academic Editor: Giovanni P. Galdi

Copyright (c) 2014 O. S. Onma et al. This is an open access article distributed under the Creative Commons Attribution License, which permits unrestricted use, distribution, and reproduction in any medium, provided the original work is properly cited.

\begin{abstract}
We propose novel controllers for stabilization and tracking of chaotic and hyperchaotic Lorenz systems using extended backstepping techniques. Based on the proposed approach, generalized weighted controllers were designed to control chaotic behaviour as well as to achieve synchronization in chaotic and hyperchaotic Lorenz systems. The effectiveness and feasibility of the proposed weighted controllers were verified numerically and showed their robustness against noise.
\end{abstract}

\section{Introduction}

Chaos theory has found application in many areas of studies; these include mathematics, physics, biology, engineering, economics, and politics [1-3]. One of the most successful applications of chaos theory has been in ecology, where dynamical systems have been used to show how population growth under density dependence can lead to chaotic dynamics. Chaos theory is also currently being applied to medical studies of epilepsy, specifically to the prediction of seemingly random seizures by observing initial conditions [4]. Furthermore, a related field of physics called quantum chaos theory investigates the relationship between chaos and quantum mechanics [5]. In addition, another field called relativistic chaos [6] has emerged to describe systems that follow the laws of general relativity.

Chaotic phenomenon could be beneficial in some applications; however, it is undesirable in many engineering and other physical applications and should therefore be controlled in order to improve the system performance. Chaos control is concerned with using some designed control inputs to modify the characteristics of a parameterized nonlinear system so that the system becomes stable at a chosen position or tracks a desired trajectory [7]. Several techniques have been deviced for chaos control but mostly are for development of two basic approaches: the OGY (Ott, Grebogi, and Yorke) method and Pyragas continuous control. Both methods require a previous determination of the unstable periodic orbits of the chaotic system before the controlling algorithm can be designed. Experimental control of chaos by one or both of these methods has been achieved in a variety of systems, including turbulent fluids, oscillating chemical reactions, magneto-mechanical oscillators, and cardiac tissues [8].

Since the idea of synchronization of chaotic systems was proposed by Pecora and Carroll in 1990, [9], chaos synchronization has received an increasing attention due to its theoretical challenge and its potential applications in secure communications, chemical reactions, biological systems, information science, and plasma technologies [10]. Chaos synchronization involves the coupling or forcing of two systems so that both systems achieve identical dynamics asymptotically with time. A wide variety of approaches have been proposed to achieve chaos synchronization in the coupled/forced chaotic systems such as linear state error feedback method [11], time-delay feedback method [12], active control approach [13, 14], impulsive method [15], adaptive control $[16,17]$, and backstepping approach [18]. The backstepping approach has been widely applied to achieve synchronization in chaotic and hyperchaotic systems. It has the ability to achieve global stability tracking transient performance for a board class of strict-feedback nonlinear system (see [7] and the references therein). Also the method requires less control 


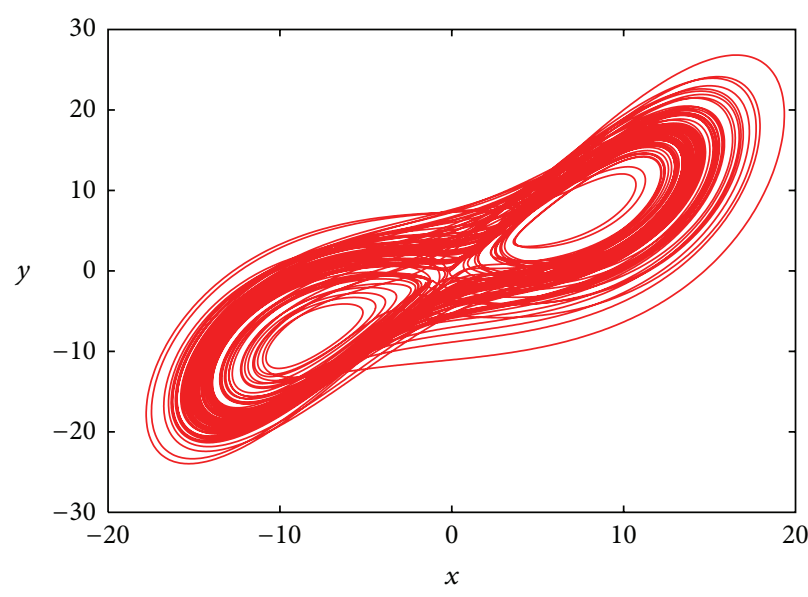

(a)

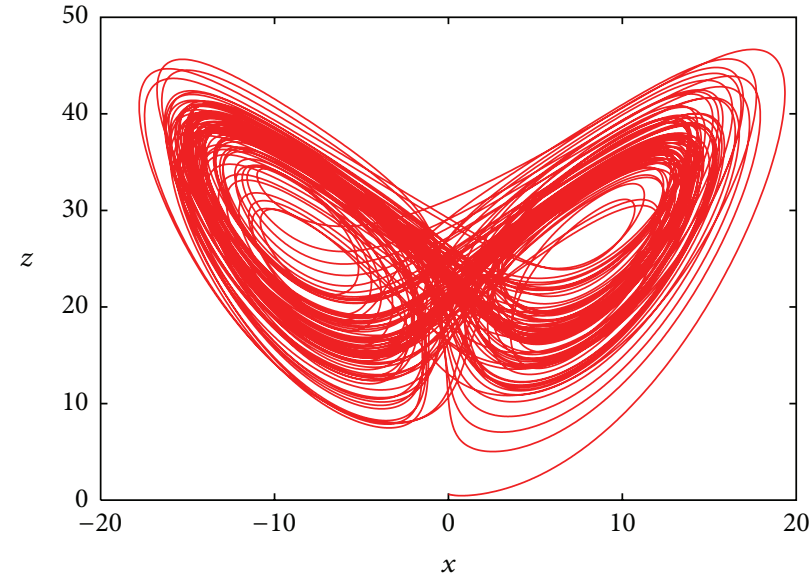

(b)

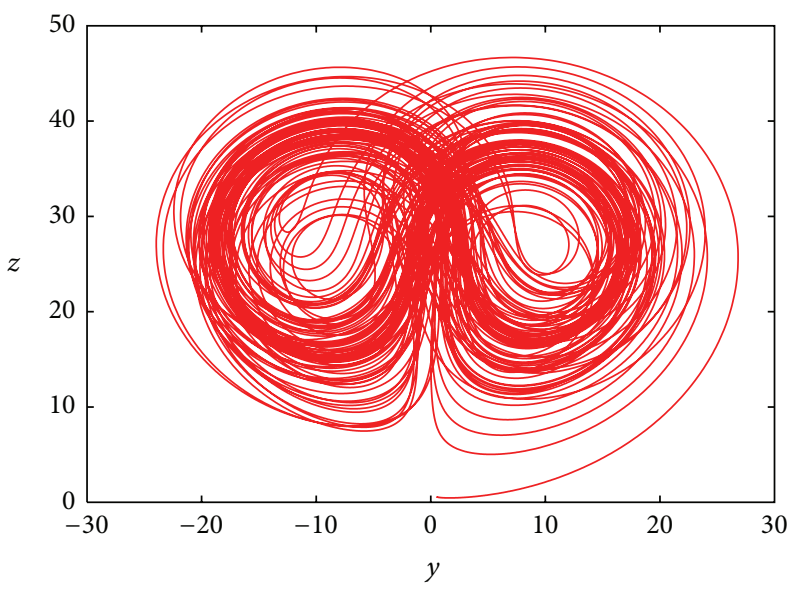

(c)

FIGURE 1: Phase portraits of chaotic attractor of Lorenz system with the parameter values $a=10, b=28$, and $c=-8 / 3$.

effort in comparison to the differential geometric method [19]. However, control function designed via this method has been shown to be difficult for practical implementation because of its complexity and high signal strength.

In order to reduce the complexity in controllers and energy consumption, the present work is focused on the design of generalized weighted controllers for stabilization and tracking of chaotic and hyperchaotic systems using extended backstepping method. Based on this approach, controller complexity is minimized and controller is singularity free from nonlinear quadratic terms $[7,11]$. The proposed approach is suitable for practical implementation and this shall be a focus in future work.

To the best of our knowledge, this approach has not been used to control as well as synchronize chaotic and hyperchaotic Lorenz systems. Also, the approach may be suitable for practical implementation in some real systems.

\section{Systems Description}

In this work, two nonlinear systems are studied, namely, chaotic Lorenz system and hyperchaotic Lorenz system. In the first instance, we consider the chaotic Lorenz system of the form

$$
\begin{aligned}
& \dot{x}=a(y-x) \\
& \dot{y}=b x-y-x z \\
& \dot{z}=-c x+x y,
\end{aligned}
$$

where $a, b$, and $c$ are the constant parameters and $x, y$, and $z$ are the state variables of the system. The dynamics of system (1) are chaotic for parameter values with the chaotic attractor shown in Figure 1.

The second system that we studied is the hyperchaotic Lorenz system describable by the following differential equations:

$$
\begin{aligned}
& \dot{x}=a(y-x) \\
& \dot{y}=b x-y-x z+w \\
& \dot{z}=-c x+x y \\
& \dot{w}=k_{1} x+k_{2} y,
\end{aligned}
$$




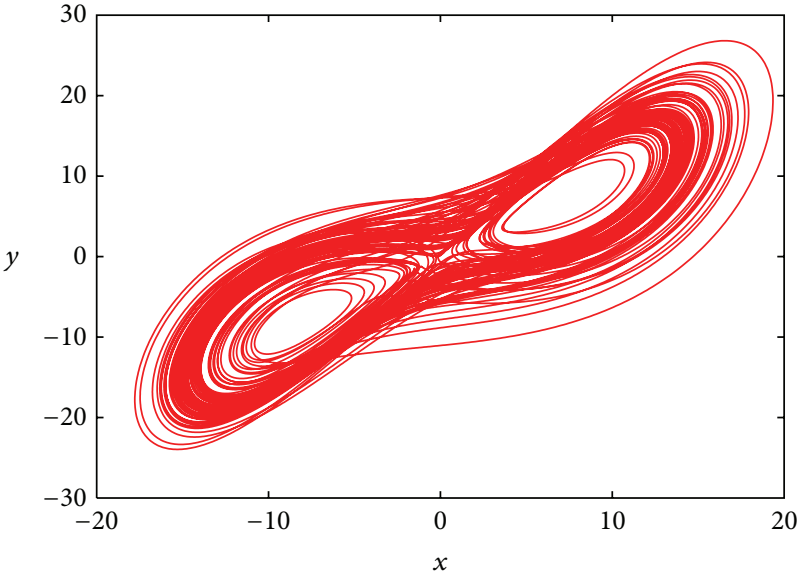

(a)

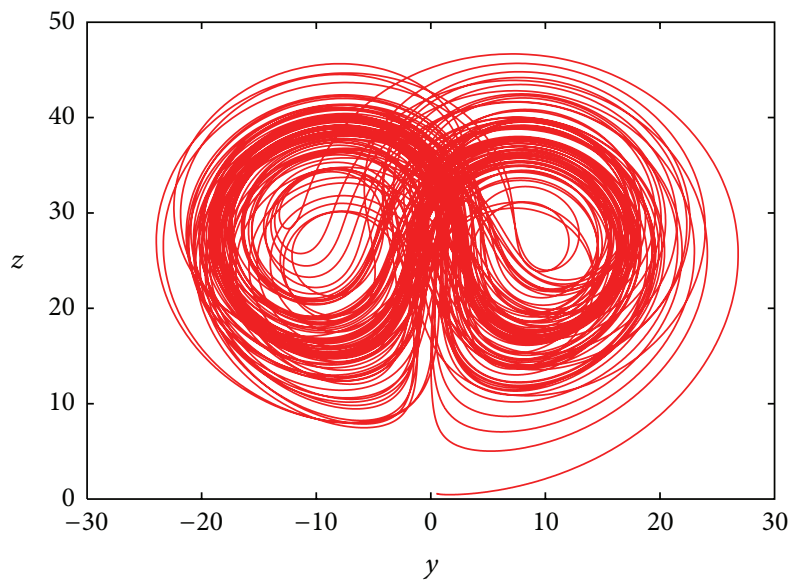

(c)

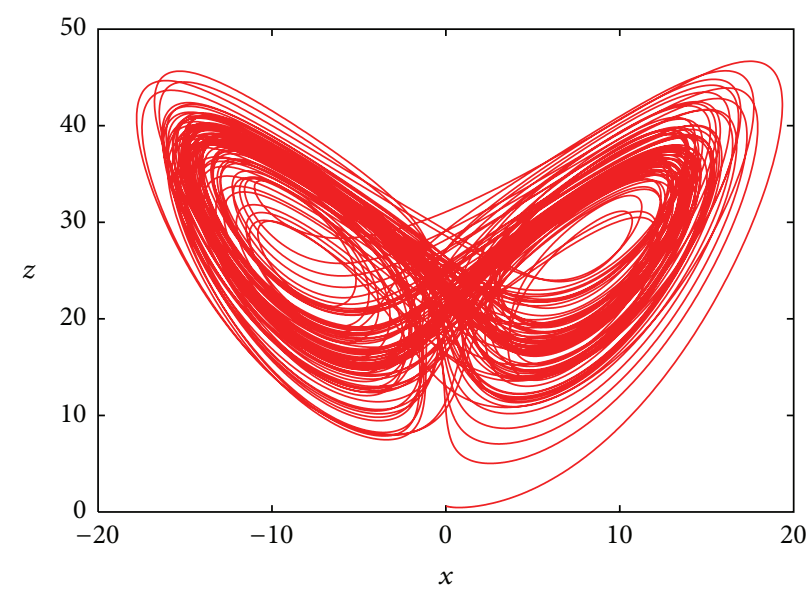

(b)

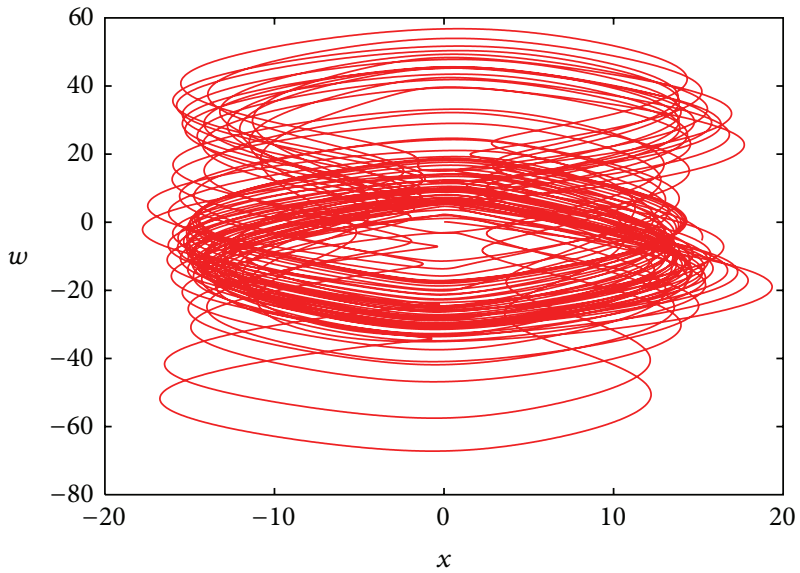

(d)

FIGURE 2: Phase portraits of the attractor of the new hyperchaotic Lorenz system with the parameter values $a=10, b=28, c=8 / 3, k_{1}=-9.3$, and $k_{2}=1$.

where $a, b, c, k_{1}$, and $k_{2}$ are the control parameters and $x, y, z$, and $w$ are the state variables of the system under investigation. The hyperchaotic system (2) is obtained by introducing a nonlinear controller to the second equation of system (1). System (2) exhibits hyperchaotic dynamics for the parameter values $a=10, b=28, c=-8 / 3, k_{1}=-9.3$, and $k_{2}=1$ with the phase portrait of the hyperchaotic attractor shown in Figure 2.

\section{Extended Controller for Tracking Control of Chaotic System}

3.1. Design of Recursive Backstepping Controller. Let us recast system (1) as follows:

$$
\begin{aligned}
\dot{x} & =a(y-x)+u_{1}(t) \\
\dot{y} & =b x-y-x z+u_{2}(t) \\
\dot{z} & =-c x+x y+u_{3}(t),
\end{aligned}
$$

where $u_{i}(t), i=1,2,3$, are the control inputs to be determined such that the state variables $x, y$, and $z$ of system (3) can take the desired values $x_{d}, y_{d}$, and $z_{d}$, respectively. We define the error states between the state variable and the desired values as

$$
\begin{aligned}
& e_{x}=x-x_{d} \\
& e_{y}=y-y_{d} \\
& e_{y}=z-z_{d} .
\end{aligned}
$$

To design general control functions $u_{i}(t), I=1,2,3$, that can control (3) to track any trajectory $f(t)$ that is a smooth function of time, we let

$$
\begin{aligned}
& x_{d}=f(t) \\
& y_{d}=c_{1} e_{x} \\
& z_{d}=c_{2} e_{x}+c_{3} e_{y},
\end{aligned}
$$


where $c_{i}, i=1,2,3$, are the arbitrary control parameter to be chosen appropriately. By substituting (5) into (4) and differentiating the resulting equation, we have

$$
\begin{aligned}
\dot{e}_{x}= & a\left(e_{y}+c_{1} e_{x}-e_{x}-f(t)\right)-\dot{f}(t)+u_{1}(t) \\
\dot{e}_{y}= & \left(e_{x}+f(t)\right)\left(b-\left(e_{z}+c_{2} e_{x}+c_{3} e_{y}\right)\right) \\
& -e_{y}-c_{1} e_{x}-c_{1} \dot{e}_{x}+u_{2}(t) \\
\dot{e}_{z}= & -c\left(e_{z}+c_{2} e_{x}+c_{3} e_{y}\right) \\
& +\left(e_{x}+f(t)\right)\left(e_{y}+c_{1} e_{x}\right)+u_{3}(t) .
\end{aligned}
$$

To stabilize the error system (6), we consider a Lyapunov function of the form

$$
V=\frac{1}{2}\left(k_{x} e_{x}^{2}+k_{y} e_{y}^{2}+k_{z} e_{z}^{2}\right)
$$

where $k_{x}, k_{y}$, and $k_{z}$ are positive constants coefficients and obtain its time derivative as

$$
\dot{V}=k_{x} e_{x} \dot{e}_{x}+k_{y} e_{y} e_{y}+\dot{k}_{z} e_{z} \dot{e}_{z}
$$

To satisfy the condition for asymptotic stability of the error dynamics (6) necessary for tracking, we substitute (6) into (8) and choose $u_{i}(t)(i=1,2,3)$ such that $\dot{V}=-\sum k_{i} e_{i}^{2}<0$, $i=x, y$, and $z$, as follows:

$$
\begin{aligned}
& u_{1}(t)=\dot{f}(t)-a\left(e_{y}+c_{1} \dot{e}_{x}-e_{x}-f(t)\right)-e_{x} \\
& u_{2}(t)=c_{1} e_{x}-\left(e_{x}+f(t)\right)\left(b-\left(e_{z}+c_{2} e_{x}+c_{3} e_{y}\right)\right) \\
& u_{3}(t)=c\left(e_{z}+c_{2} e_{x}+c_{3} e_{y}\right)-\left(e_{x}+f(t)\right)\left(e_{y}+c_{1} e_{x}\right)-e_{z} .
\end{aligned}
$$

We observed that system (3) is effectively controlled with $c_{1}=$ $c_{3}=1$ and $c_{2}=0$ which simplify the controllers in (9) to

$$
\begin{aligned}
& u_{1}(t)=\dot{f}(t)-a\left(\dot{e}_{y}-f(t)\right)-e_{x} \\
& u_{2}(t)=e_{x}-\left(e_{x}+f(t)\right)\left(b-\left(e_{z}+e_{y}\right)\right) \\
& u_{3}(t)=c\left(e_{z}+c_{2} e_{x}+c_{3} e_{y}\right)-\left(e_{x}+f(t)\right)\left(e_{y}+c_{1} e_{x}\right)-e_{z} .
\end{aligned}
$$

By introducing weights to the above controllers, we obtain the following:

$$
\begin{aligned}
& u_{1}(t)= {\left[\dot{f}(t)-a\left(\dot{e}_{y}-f(t)\right)-e_{x}\right] \varepsilon_{1} } \\
& u_{2}(t)=\left[e_{x}-\left(e_{x}+f(t)\right)\left(b-\left(e_{z}+e_{y}\right)\right)\right] \varepsilon_{2} \\
& u_{3}(t)=\left[c\left(e_{z}+c_{2} e_{x}+c_{3} e_{y}\right)\right. \\
&\left.-\left(e_{x}+f(t)\right)\left(e_{y}+c_{1} e_{x}\right)-e_{z}\right] \varepsilon_{3},
\end{aligned}
$$

where $\varepsilon_{i}, i=1,2,3$, are the weights added to the usual controllers.
3.2. Numerical Simulation. Using the fourth-order Runge-Kutta algorithm with initial conditions $(x, y, z)=$ $(-0.1,0.2,6.0)$, a time step of 0.005 , and fixing the parameter values of $a, b$, and $c$ as in Figure 1 to ensure chaotic dynamics of the state variables, we solved system (3) with the controllers $u_{i}(t), i=1,2,3$, as defined in (10) and (11). The results obtained show that the state variables move chaotically with time when the controller is switched off and when the controller is activated at time $t=50$ the state variables are controlled to track the desired functions $f(t)=35 \cos 0.57 t$. The result is shown in Figure 3 . With the value of $\varepsilon_{i}=0.5$ (Figure 4 ) where $i=1,2,3$ we observed that the controllers (10) and (11) are capable of controlling the dynamics of the chaotic system (3) to track any desired smooth function, $f(t)$, and to stabilize it at any position $P$ (case $f(t)=P$ ) and when $P=0$ the system becomes stabilized at the equilibrium point $(0,0,0)$. The results in Figure 4 showed that the proposed extended backstepping techniques reduced the control strength and complexity of the designed controllers by $50 \%$, thereby producing economic controllers with low energy consumption which are suitable for practical implementation.

\section{Extended Controller for Tracking Control of the Hyperchaotic System}

4.1. Design of the Recursive Backstepping Controller. In order to design controllers for tracking control of hyperchaotic Lorenz system we recast (2) as follows:

$$
\begin{aligned}
& \dot{x}=a(y-x)+u_{1}(t) \\
& \dot{y}=b x-y-x z+w+u_{2}(t) \\
& \dot{z}=-c x+x y+u_{3}(t) \\
& \dot{w}=k_{1} x+k_{2} y+u_{4}(t),
\end{aligned}
$$

where $u_{i}, i=1,2,3,4$, are the control inputs to be determined later such that the state variables $x, y, z$, and $w$ of system (13) can take the desired values $x_{d}, y_{d}, z_{d}$, and $w_{d}$, respectively. We define the error states between the state variable and the desired values as

$$
\begin{aligned}
& e_{x}=x-x_{d} \\
& e_{y}=y-y_{d} \\
& e_{z}=z-z_{d} \\
& e_{w}=w-w_{d} .
\end{aligned}
$$

To design general control functions, $u_{i}(t)$, that can control system (12) to track any trajectory $f(t)$ that is a smooth function of time, we let

$$
\begin{aligned}
& x_{d}=f(t) \\
& y_{d}=c_{1} e_{x} \\
& z_{d}=c_{2} e_{x}+c_{3} e_{y} \\
& w_{d}=c_{4} e_{x}+c_{5} e_{y}+c_{6} e_{z},
\end{aligned}
$$




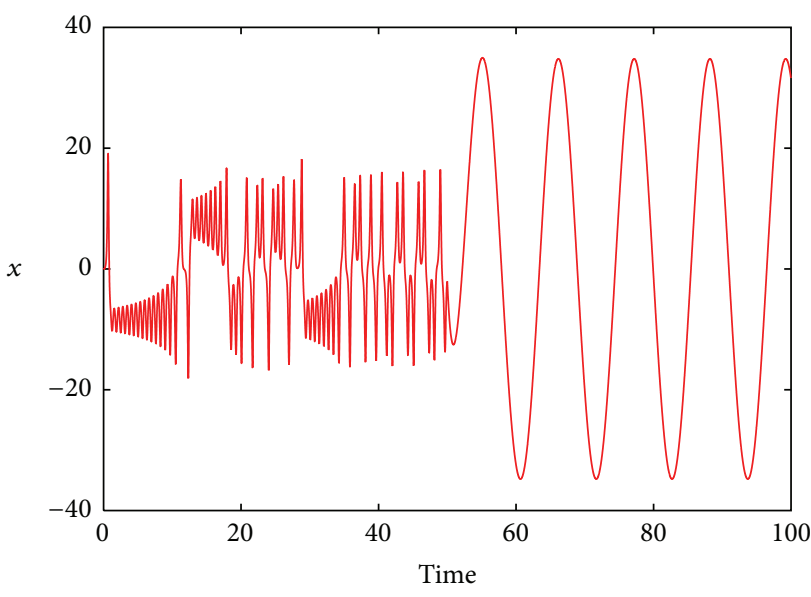

(a)

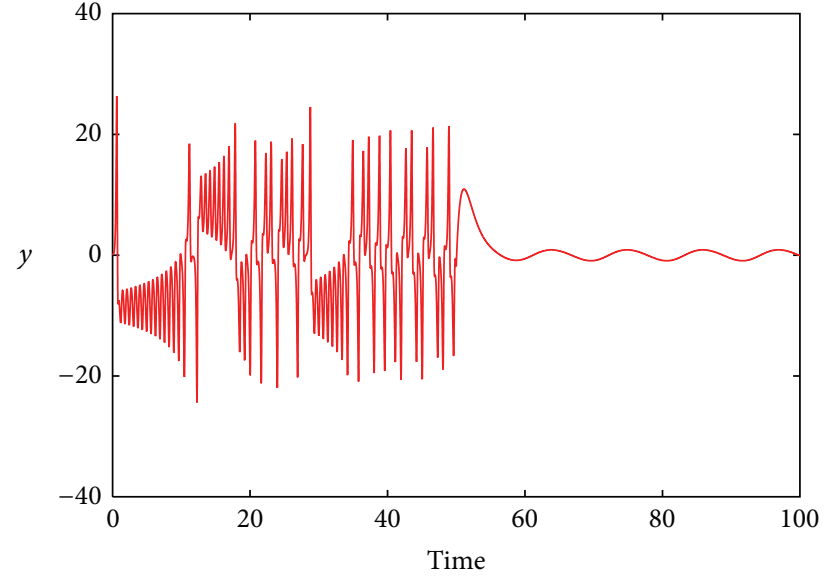

(b)

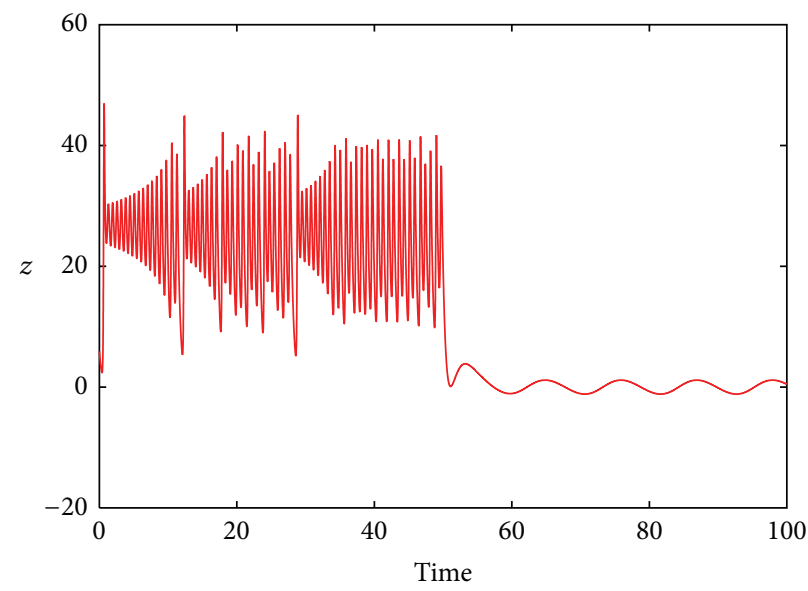

(c)

Figure 3: Recursive backstepping tracking control of chaotic Lorenz system for $f(t)=35 \cos 0.57 t$ when the controller is activated at $t=50$. The parameters of the system are as shown in Figure 1.

where $c_{i}(i=1,2, \ldots 6)$ are arbitrary control parameters to be chosen appropriately. Substitution of (14) into (13) and carrying out some algebraic manipulations yield

$$
\begin{aligned}
\dot{e}_{x}= & a\left(e_{y}+c_{1} e_{x}-e_{x}-f(t)\right)-\dot{f}(t)+u_{1}(t) \\
\dot{e}_{y}= & \left(e_{x}+f(t)\right)\left(b-\left(e_{z}+c_{2} e_{x}+c_{3} e_{y}\right)\right) \\
& +e_{w}+c_{4} e_{x}+c_{5} \dot{e}_{y}+c_{6} e_{z}-e_{y}-c_{1} \dot{e}_{x}-c_{1} \dot{e}_{x}+u_{2}(t) \\
\dot{e}_{z}= & -c\left(e_{z}+c_{2} e_{x}+c_{3} e_{y}\right)+\left(e_{x}+f(t)\right)\left(e_{y}+c_{1} e_{x}\right) \\
& -c_{2} \dot{e}_{x}-c_{3} \dot{e}_{y}+u_{3}(t) \\
\dot{e}_{w}= & k_{1}\left(e_{x}+f(t)\right)+k_{2}\left(e_{y}+c_{1} e_{x}\right) \\
& -c_{4} \dot{e}_{x}-c_{5} \dot{e}_{y}-c_{6} \dot{e}_{z}+u_{4}(t) .
\end{aligned}
$$

To stabilize the error system (15), we consider a Lyapunov function of the form

$$
V=\frac{1}{2}\left(k_{x} e_{x}^{2}+k_{y} e_{y}^{2}+k_{z} e_{z}^{2}+k_{w} e_{w}^{2}\right)
$$

where $k_{x}, k_{y}, k_{z}$ and $k_{w}$, are positive constants coefficients and obtain its time derivative as

$$
\dot{V}=k_{x} e_{x} \dot{e}_{x}+k_{y} e_{y} \dot{e}_{y}+k_{z} e_{z} \dot{e}_{z}+k_{w} e_{w} \dot{e}_{w}
$$

To satisfy the condition for asymptotic stability of the error dynamics (6) necessary for tracking, we substitute (15) into 


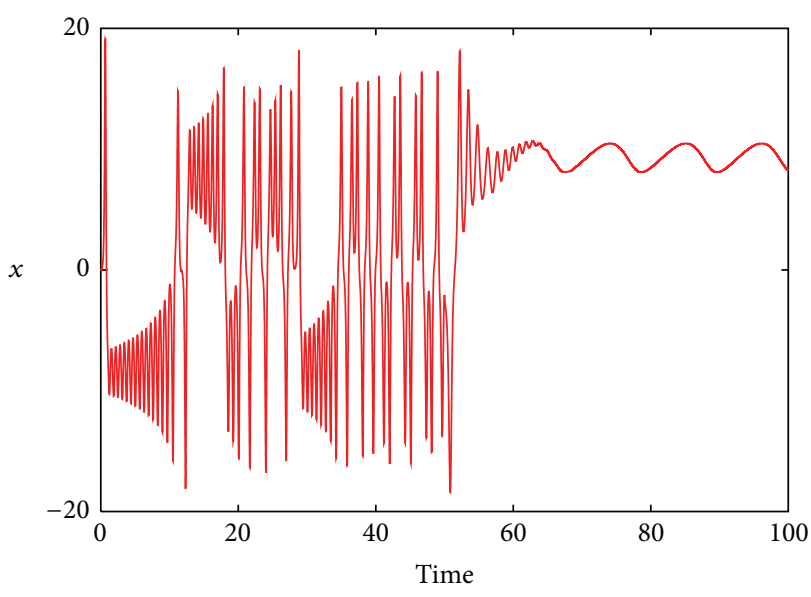

(a)

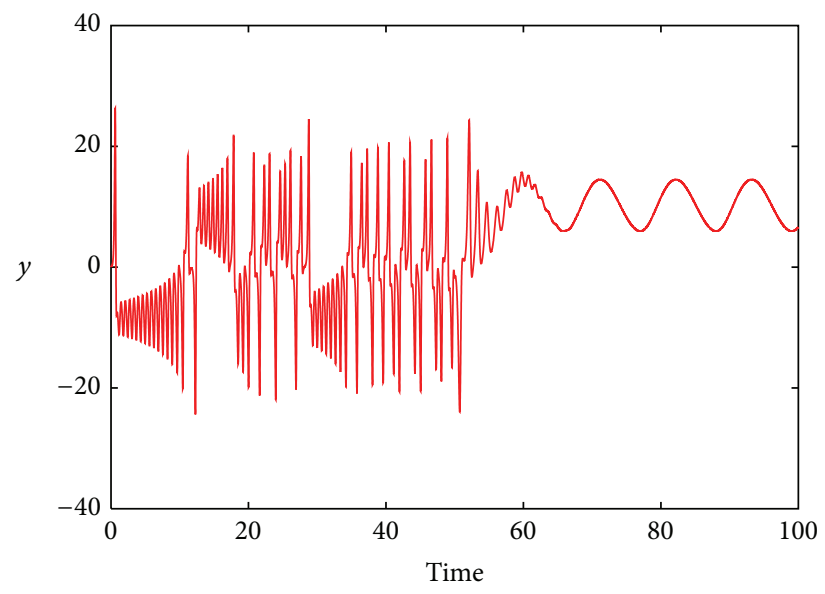

(b)

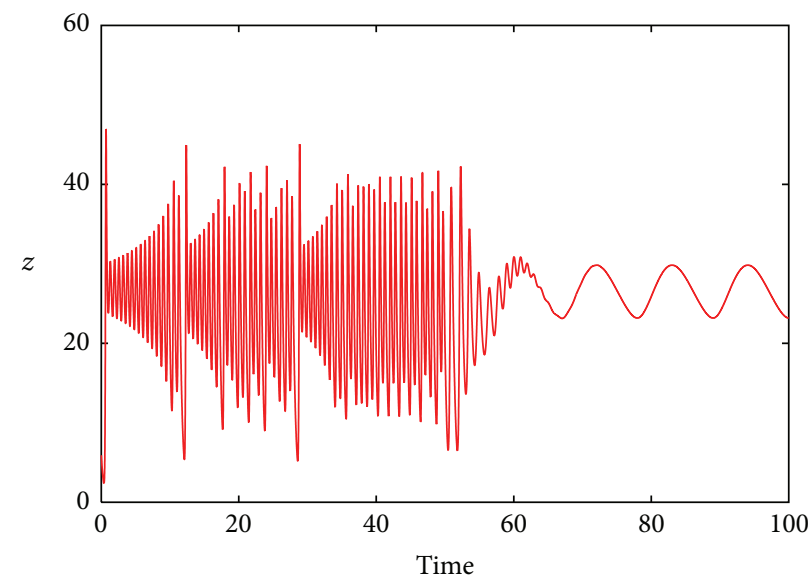

(c)

FIGURE 4: Extended recursive backstepping tracking control of chaotic Lorenz system for $f(t)=35$ cos $0.57 t$ when the controller is activated at $t=50$. The parameters of the system are as shown in Figure 1 with $\varepsilon_{i}=0.5$, where $i=1,2,3$.

(17) and choose $u_{i}(t)(i=1,2,3,4)$ such that $\dot{V}=-\sum k_{i} e_{i}^{2}<$ $0, i=x, y, z$, and $w$, as follows:

$$
\begin{aligned}
u_{1}(t)= & \dot{f}(t)-a\left(e_{y}+c_{1} e_{x}-e_{x}-f(t)\right)-\dot{e}_{x} \\
u_{2}(t)= & -\left(e_{x}+f(t)\right)\left(b-\left(e_{z}+c_{2} e_{x}+c_{3} e_{y}\right)\right) \\
& -e_{w}-c_{4} e_{x}-c_{5} \dot{e}_{y}-c_{6} e_{z}+c_{1} e_{x} \\
u_{3}= & c\left(e_{z}+c_{2} e_{x}+c_{3} e_{y}\right) \\
& -\left(e_{x}+f(t)\right)\left(e_{y}+c_{1} e_{x}\right)-e_{z} \\
u_{4}= & -k_{1}\left(e_{x}+f(t)\right)-k_{2}\left(e_{y}+c_{1} e_{x}\right)-e_{w} .
\end{aligned}
$$

We have observed from the results of computations that system (12) is effectively controlled with only $c_{1}=1$ and so we set $c_{2}=c_{3}=c_{4}=c_{5}=c_{6}=0$ which simplify the controllers (18) to

$$
\begin{aligned}
u_{1}(t) & =\dot{f}(t)-a\left(\dot{e}_{y}-f(t)\right)-e_{x} \\
u_{2}(t) & =e_{x}-\left(e_{x}+f(t)\right)\left(b-e_{z}\right)-e_{w} \\
u_{3} & =c e_{z}-\left(e_{x}+f(t)\right)\left(e_{y}+e_{x}\right)-e_{z} \\
u_{4} & =-k_{1}\left(e_{x}+f(t)\right)-k_{2}\left(e_{y}+e_{x}\right)-e_{w} .
\end{aligned}
$$

By introducing weights to the above controllers, we obtain the following:

$$
\begin{aligned}
u_{1}(t) & =\left[\dot{f}(t)-a\left(\dot{e}_{y}-f(t)\right)-e_{x}\right] \varepsilon_{1} \\
u_{2}(t) & =\left[e_{x}-\left(e_{x}+f(t)\right)\left(b-e_{z}\right)-e_{w}\right] \varepsilon_{2} \\
u_{3} & =\left[c e_{z}-\left(e_{x}+f(t)\right)\left(e_{y}+e_{x}\right)-e_{z}\right] \varepsilon_{3} \\
u_{4} & =\left[-k_{1}\left(e_{x}+f(t)\right)-k_{2}\left(e_{y}+e_{x}\right)-e_{w}\right] \varepsilon_{4},
\end{aligned}
$$




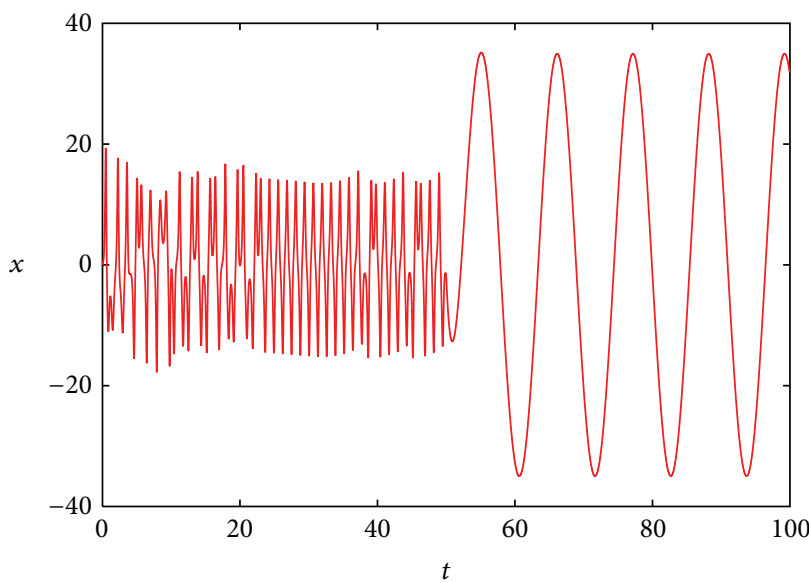

(a)

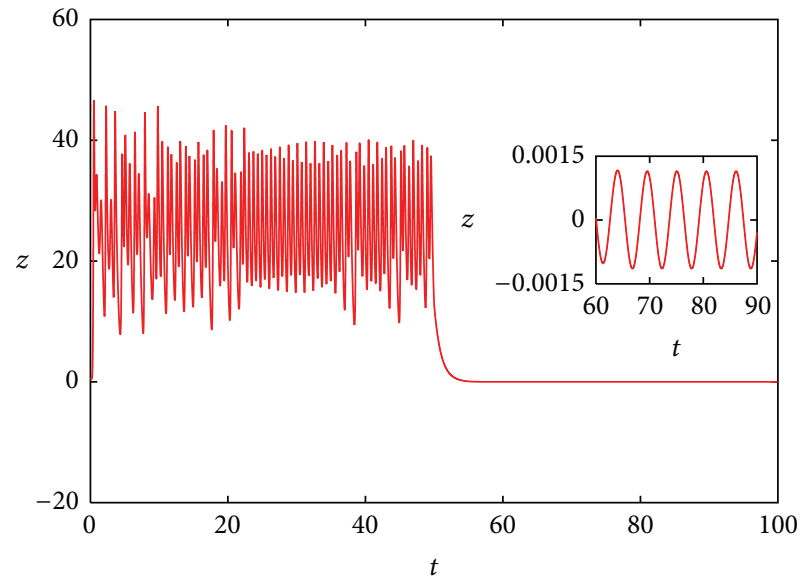

(c)

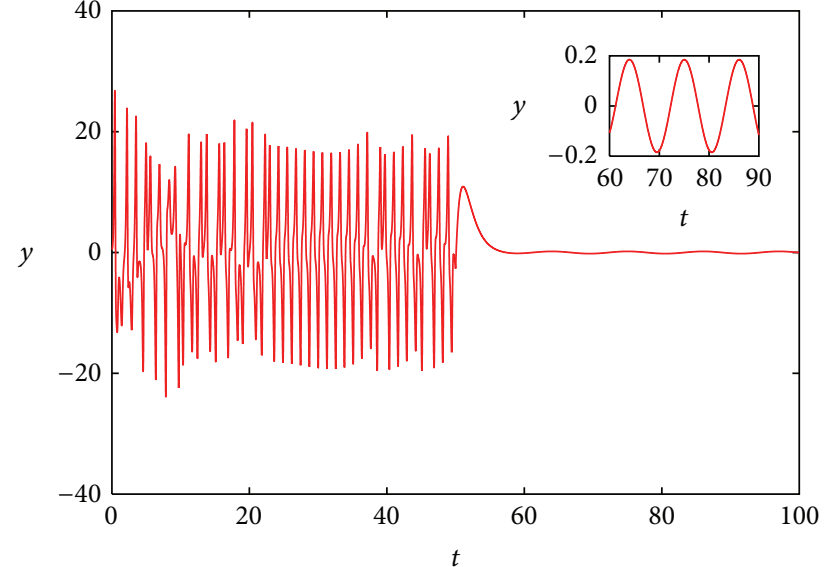

(b)

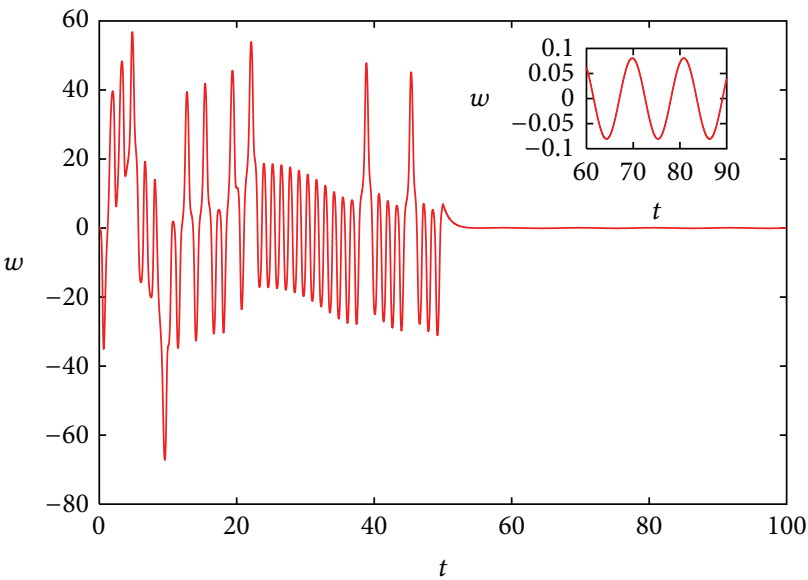

(d)

FIGURE 5: Recursive backstepping tracking control of hyperchaotic Lorenz system for $f(t)=35 \cos 0.57 t$ when the controller is activated at $t=50$. The parameters of the system are as shown in Figure 2.

where $\varepsilon_{i}, i=1,2,3,4$, are the weights added to the usual controllers.

4.2. Numerical Simulation. Using the fourth-order RungeKutta algorithm with initial conditions $(x, y, z, w)=$ $(0.0,0.5,0.6,0.1)$, a time step of 0.001 , and fixing the parameter values of $a, b, c, k_{1}$, and $k_{2}$ as in Figure 2 to ensure hyperchaotic dynamics of the state variables, we solved system (12) with the controllers $u_{i}(t), i=1,2,3,4$, as defined in (19) and (20). The results obtained show that the state variables move hyperchaotically with time when the controller is deactivated and when the controller is switched on at time $t=50$ the state variables are controlled to track the desired functions $f(t)=$ $35 \cos 0.57 t$ as shown in Figure 5. With the choice of the weights, $\varepsilon_{1}=\varepsilon_{2}=0.7$ and $\varepsilon_{3}=\varepsilon_{4}=0.8$ in (20), we displayed the result of our simulation in Figure 6. We observed that the controllers (19) and (20) are capable of controlling the dynamics of the hyperchaotic system (12) to track any desired smooth function, $f(t)$, and to stabilize it at any position $P($ case $f(t)=P)$ and when $P=0$ the system becomes stabilized at the equilibrium point $(0,0,0,0)$. By comparing the results in Figure 5 (recursive backstepping controllers) with those of Figure 6 (extended backstepping method), it is obvious that when the weights were added to the controllers, control strength and complexity were reduced significantly. Thus, the proposed extended backstepping controllers can produce economic controllers with low energy consumption which may be suitable for practical implementation.

\section{Extended Backstepping Synchronization of the Chaotic Systems}

5.1. Design of the Extended Backstepping Controller for Chaotic Lorenz System. In order to derive controllers for synchronization in chaotic Lorenz system, we rewrite (1) in the form

$$
\begin{aligned}
& \dot{x}_{1}=a\left(x_{2}-x_{1}\right) \\
& \dot{x}_{2}=b x_{1}-x_{2}-x_{1} x_{3} \\
& \dot{x_{3}}=-c x_{3}+x_{1} x_{2} .
\end{aligned}
$$




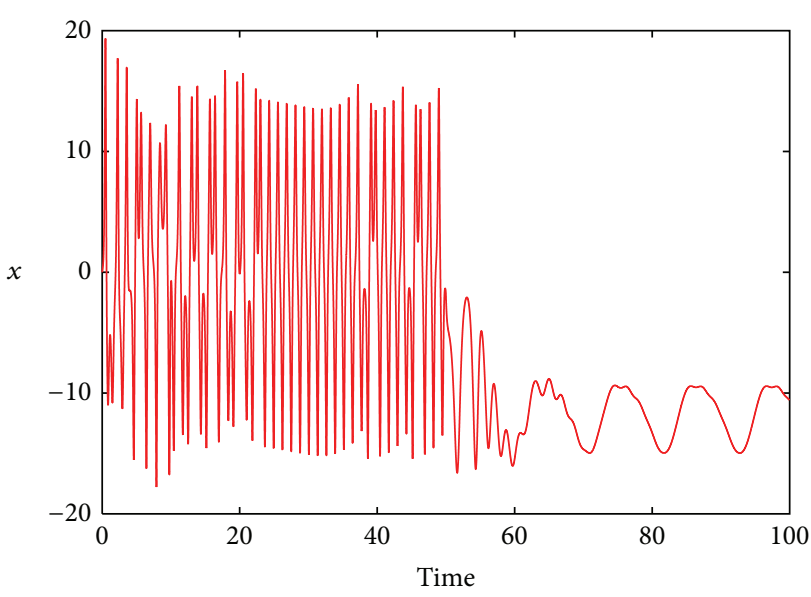

(a)

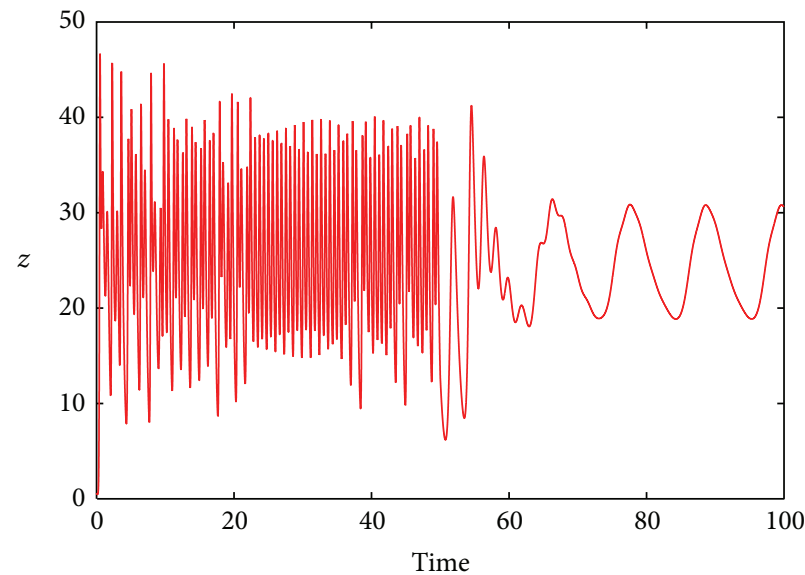

(c)

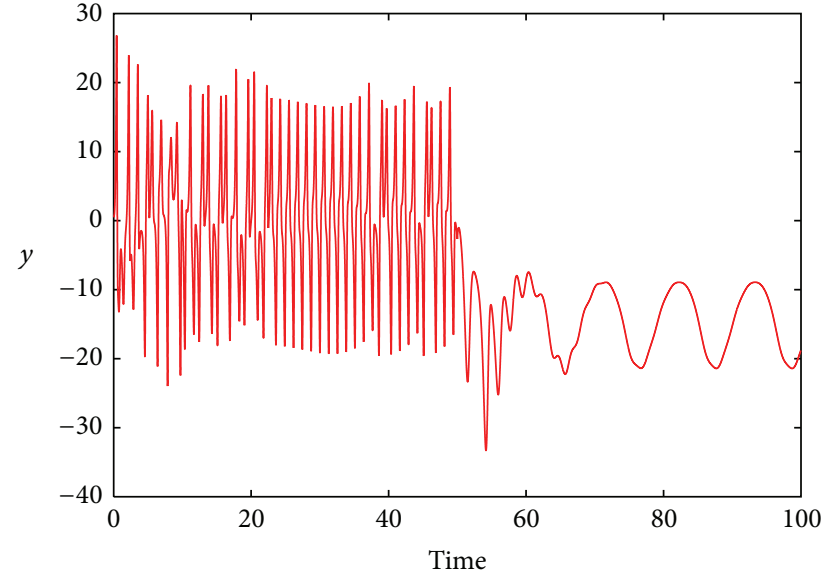

(b)

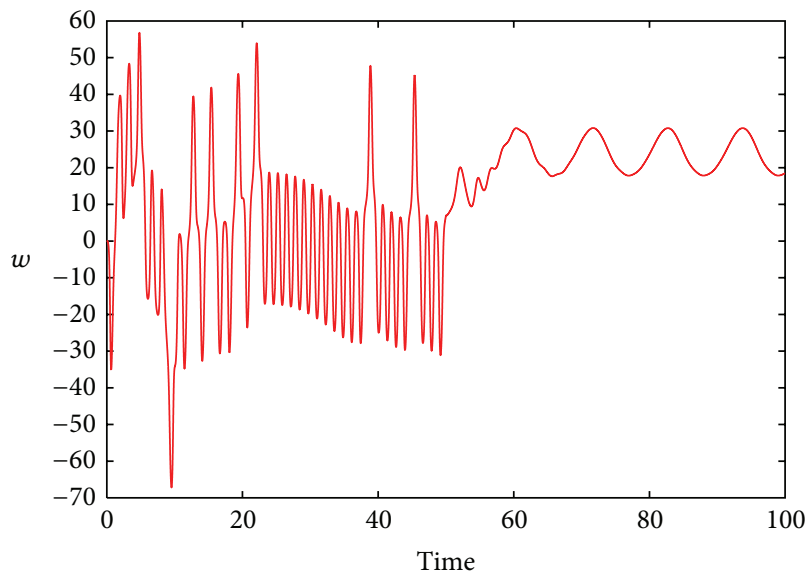

(d)

FIGURE 6: Extended recursive backstepping tracking control of hyperchaotic Lorenz system for $f(t)=35$ cos $0.57 t$ when the controller is activated at $t=50$. The parameters of the system are as shown in Figure 2 with $\varepsilon_{1}=\varepsilon_{2}=0.7$ and $\varepsilon_{3}=\varepsilon_{4}=0.8$.

Let system (21) be the drive system and let the following be the response system:

$$
\begin{aligned}
& \dot{y}_{1}=a\left(y_{2}-y_{1}\right)+u_{1}(t) \\
& \dot{y}_{2}=b y_{1}-y_{2}-y_{1} y_{3}+u_{2}(t) \\
& \dot{y}_{3}=-c y_{3}+y_{1} y_{2}+u_{3}(t),
\end{aligned}
$$

where $u_{i}, i=1,2,3$, are the control functions. Subtract (21) from (22) and use the notations

$$
e_{i}=y_{i}-x_{i}, \quad i=1,2,3 .
$$

The error system is obtained as

$$
\begin{aligned}
& \dot{e}_{1}=a\left(e_{2}-\dot{e}_{1}\right)+u_{1} \\
& \dot{e_{2}}=b e_{1}-e_{2}-\left(x_{1} e_{3}+x_{3} e_{1}+e_{1} e_{3}\right)+u_{2} \\
& \dot{e}_{3}=-c e_{3}+\left(x_{1} e_{2}+x_{2} e_{1}+e_{1} e_{2}\right)+u_{3} .
\end{aligned}
$$

The objective is to find control functions, $u_{i}(t)$, that can stabilize the error states in (24) at origin. Firstly, we stabilize the first equation in (24) by regarding $e_{2}$ as a controller, choosing a Lyapunov function $V_{1}\left(e_{1}\right)=(1 / 2) e_{1}^{2}$, and differentiating it with respect to time to have

$$
\dot{V}_{1}=e_{1} \dot{e}_{1}=e_{1}\left[a\left(e_{2}-e_{1}\right)+u_{1}\right] .
$$

We estimate that the controller $e_{2}=\alpha_{1}\left(e_{1}\right)$; then (25) can be written as; if $u_{1}=0$ and estimative function $\alpha_{1}\left(e_{1}\right)=$ 0 , then $\dot{V}_{1}=-a e_{1}^{2}$ is negative definite (since $a>0$ ), and hence the $e_{1}$ subsystem is stabilized. The error $\omega_{2}$ between $e_{2}$ and $\alpha_{1}\left(e_{1}\right)$ is

$$
\omega_{2}=e_{2}-\alpha_{1}\left(e_{1}\right)=e_{2} .
$$

Substitution of $\dot{e}_{2}$ and $e_{2}$ from (24) and (26), respectively, into the time derivative of (26) yields

$$
\dot{\omega}_{2}=\left(b-x_{3}\right) e_{1}-\omega_{2}-\left(x_{1}+e_{1}\right) e_{3}+u_{2} .
$$

We now stabilize the $\left(e_{1}, \omega_{2}\right)$ subsystem given by $(27)$ as follows. We choose a Lyapunov function $V_{2}\left(e_{1}, \omega_{2}\right)=V_{1}\left(e_{1}\right)+$ $(1 / 2) \omega_{2}^{2}$ and obtain its time derivative as

$$
V_{2}\left(e_{1}, \omega_{2}\right)=\dot{V}_{1}\left(e_{1}\right)+\omega_{2} \dot{\omega}_{2} .
$$




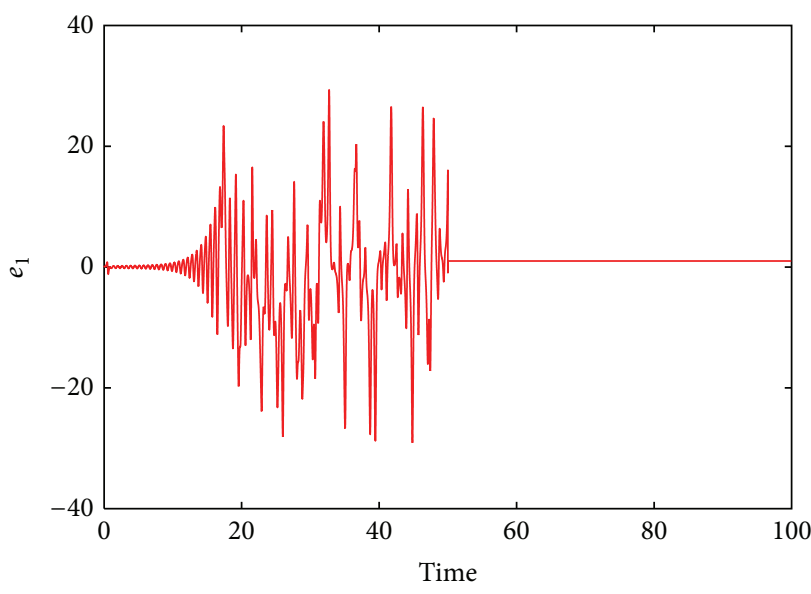

(a)

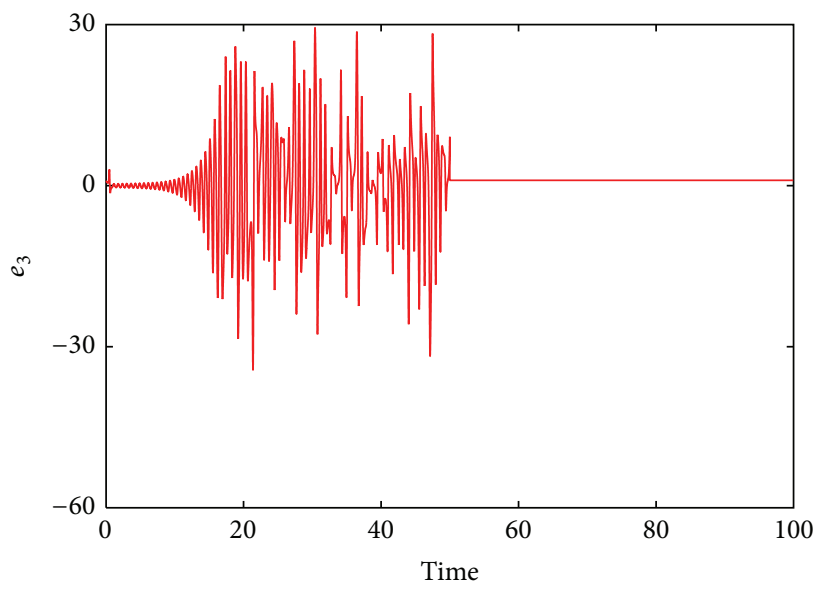

(c)

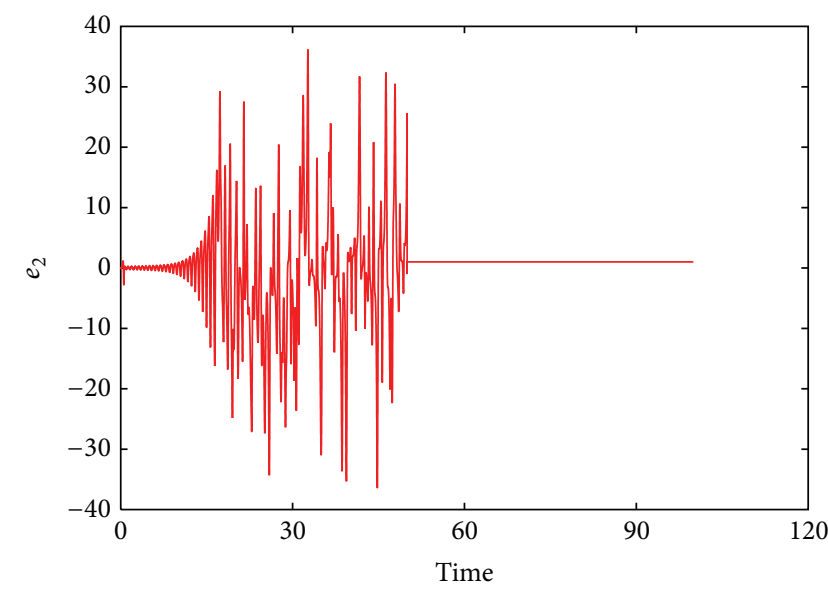

(b)

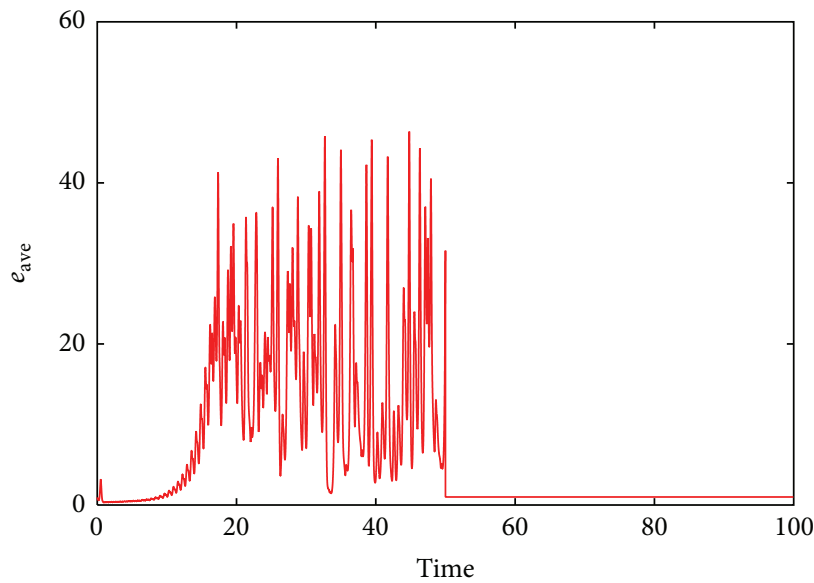

(d)

Figure 7: Error dynamics between the two chaotic Lorenz systems with the controller deactivated for $0<t<50$ and activated for $t \geq 50$. The parameters of the system are as shown in Figure 1.

From (25) and (26), we have

$$
\dot{V}_{1}\left(e_{1}\right)=-a e_{1}^{2}+a e_{1} \omega_{2} .
$$

Hence,

$$
\begin{aligned}
\dot{V}_{2}\left(e_{2}\right)= & -a e_{1}^{2}+a e_{1} \omega_{2} \\
& +\omega_{2}\left[\left(b-x_{3}\right) e_{1}-\omega_{2}-\left(x_{1}+e_{1}\right) e_{3}+u_{2}\right] .
\end{aligned}
$$

Estimating that the controller $e_{3}=\alpha_{2}\left(e_{1}, \omega_{2}\right)$, then (30) can be written as

$$
\begin{aligned}
\dot{V}_{2}= & -a e_{1}^{2}+a e_{1} \omega_{2} \\
& +\omega_{2}\left[\left(b-x_{3}\right) e_{1}-\omega_{2}-\left(x_{1}+e_{1}\right) \alpha_{2}+u_{2}\right] .
\end{aligned}
$$

If the estimative function $\alpha_{2}\left(e_{1}, \omega_{2}\right)=0$ and $u_{2}=-\left(b-x_{3}+\right.$ a) $e_{1}$, then $\dot{V}_{2}=-a e_{1}^{2}-\omega^{2}$ is negative definite (since $a, b>$ $0)$ and hence the $\left(e_{1}, \omega_{1}\right)$ subsystem is stabilized. The error between $e_{3}$ and $\alpha_{2}\left(e_{1}, \omega_{2}\right)$ is

$$
\omega_{3}=e_{3}-\alpha_{2}\left(e_{1}, \omega_{2}\right)=e_{3} \text {. }
$$

Substituting for $\dot{e}_{3}, e_{3}$, and $\omega_{2}$ from (24), (32), and (26), respectively, into time derivative of (32) yield

$$
\dot{\omega}_{3}=-c \omega_{3}+\left(x_{2}+\omega_{2}\right) e_{1}+x_{1} \omega_{2}+u_{3} .
$$

We now stabilize the $\left(e_{1}, \omega_{2}, \omega_{3}\right)$ complete system given by (26), (27), and (33) as follows. We choose a Lyapunov function $V_{3}\left(e_{1}, \omega_{2}, \omega_{3}\right)=V_{2}\left(e_{1}, \omega^{2}\right)+(1 / 2) \omega_{3}^{2}$ and obtain its time derivative as

$$
\dot{V}_{3}\left(e_{1}, \omega_{2}, \omega_{3}\right)=\dot{V}_{2}\left(e_{1}, \omega^{2}\right)+\omega_{3} \dot{\omega}_{3} .
$$

Hence,

$$
\dot{V}_{3}=-a e_{1}^{2}-\omega_{1}^{2}+\omega_{3}\left[-c \omega_{3}+\left(x_{2}+\omega_{2}\right) e_{1}+x_{1} \omega_{2}+u_{3}\right] .
$$

Estimating that $u_{3}(t)=-\left(x_{2}+\omega_{2}\right) e_{1}-x_{1} \omega_{2}$,

$$
V_{3}=-a e_{1}^{2}-\omega_{2}^{2}-c \omega_{3}^{2}<0 \quad(\text { since } a, b, c>0) .
$$




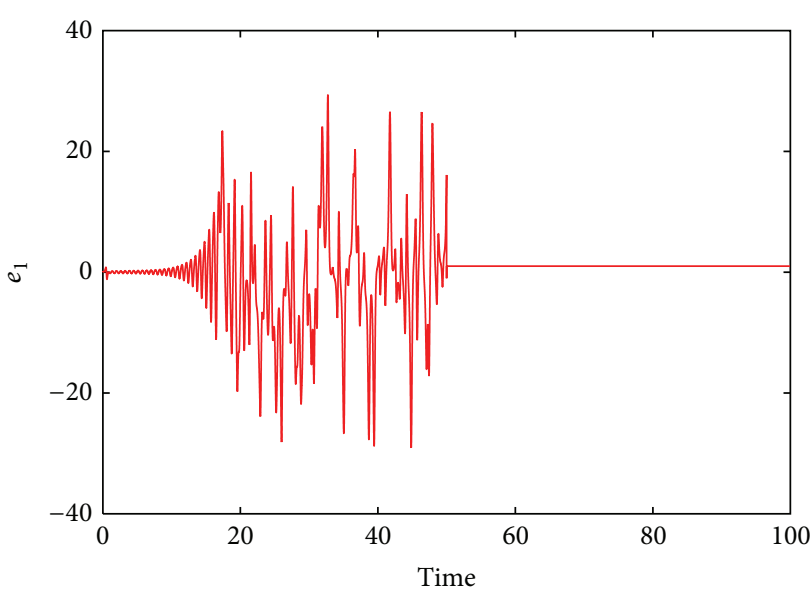

(a)

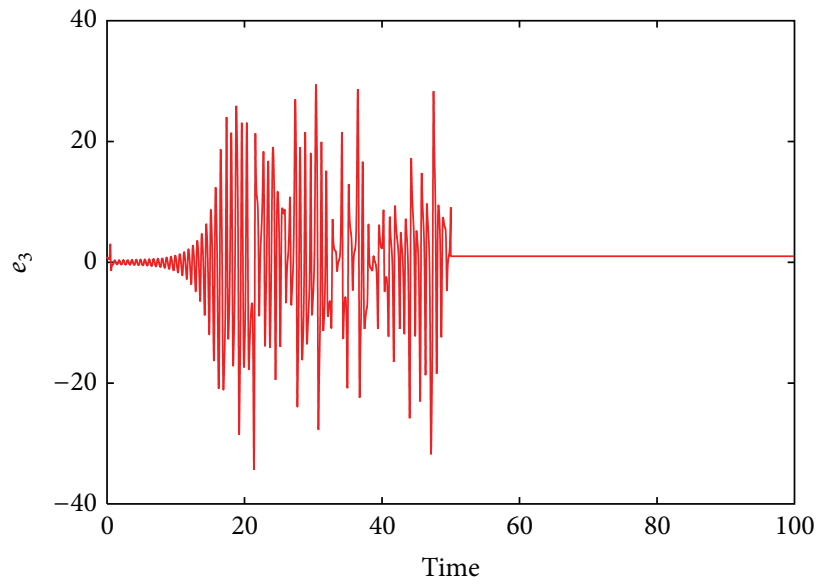

(c)

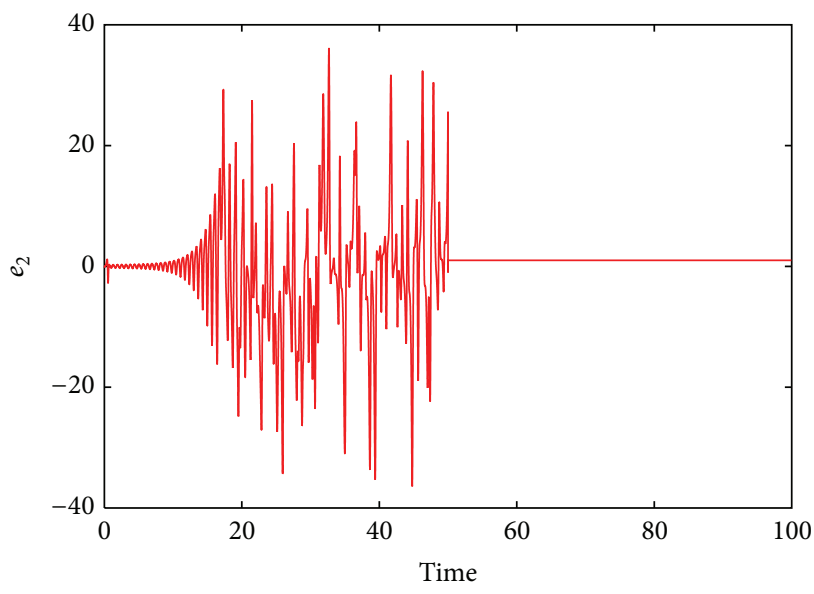

(b)

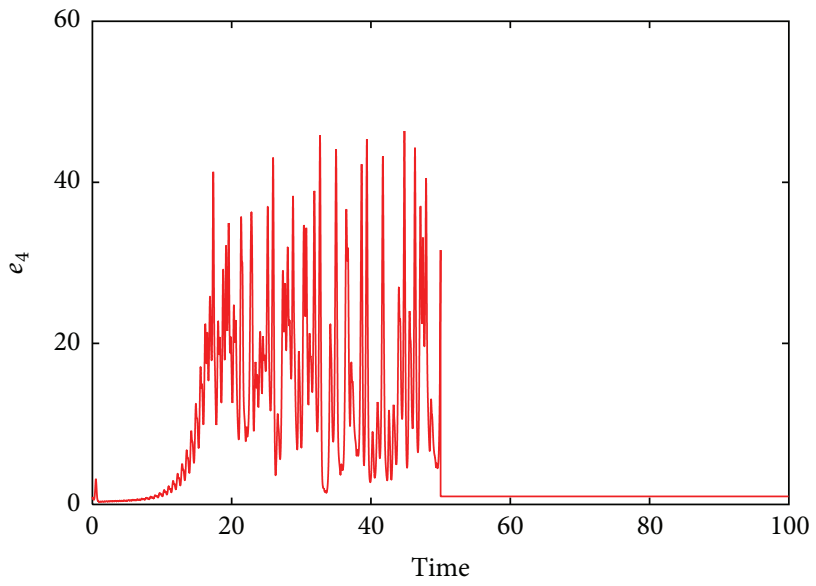

(d)

Figure 8: Error dynamics between the two chaotic Lorenz systems with the controller deactivated for $0<t<50$ and activated for $t \geq 50$. The parameters of the system are as shown in Figure 1 with $\varepsilon_{i}=0.3$, where $i=1,2,3$.

Thus the synchronization goal is achieved with the weight added to the control function:

$$
\begin{aligned}
& u_{1}(t)=0 \\
& u_{2}(t)=-\left(b-x_{3}+a\right) e_{1} \\
& u_{3}(t)=-\left(x_{2}+\omega_{2}\right) e_{1}-x_{1} \omega_{2} .
\end{aligned}
$$

By introducing weights to the above controllers, the extended backstepping controllers are obtained as follows:

$$
\begin{aligned}
& u_{1}(t)=[0] \varepsilon_{1} \\
& u_{2}(t)=\left[-\left(b-x_{3}+a\right) e_{1}\right] \varepsilon_{2} \\
& u_{3}(t)=\left[-\left(x_{2}+\omega_{2}\right) e_{1}-x_{1} \omega_{2}\right] \varepsilon_{3} .
\end{aligned}
$$

5.2. Numerical Simulation. Using the fourth-order RungeKutta routine with initial conditions $\left(x_{1}, x_{2}, x_{3}\right)=(0.8-$ $0.8,-1.0),\left(y_{1}, y_{2}, y_{3}\right)=(1.0,-1.0,-0.1)$, a time step of 0.001 , and fixing the parameter values as in Figure 1 to ensure chaotic dynamics of the state variables, we solved systems (21) and (22) with the control function as defined in (37) and (38). The result obtained shows that the error state variable moves chaotically with time when the controllers are switched off and when the controllers are switched on at $t=50$ as shown in Figures 7 and 8 the error state variables converge to zero, thereby guaranteeing the synchronization of systems (21) and (22). This is confirmed by the synchronization quality $e$ given by

$$
e=\sqrt{e_{1}^{2}+e_{2}^{2}+e_{3}^{2}} .
$$

Remarkably, we notice that the control strength and its complexity are reduced by about $70 \%$ when the proposed extended backstepping techniques is applied (Figure 8) compared with the usual backstepping approach. Thus, the new approach proposed in this study produces economic controllers with low energy consumption which may be of vital importance for practical application. 


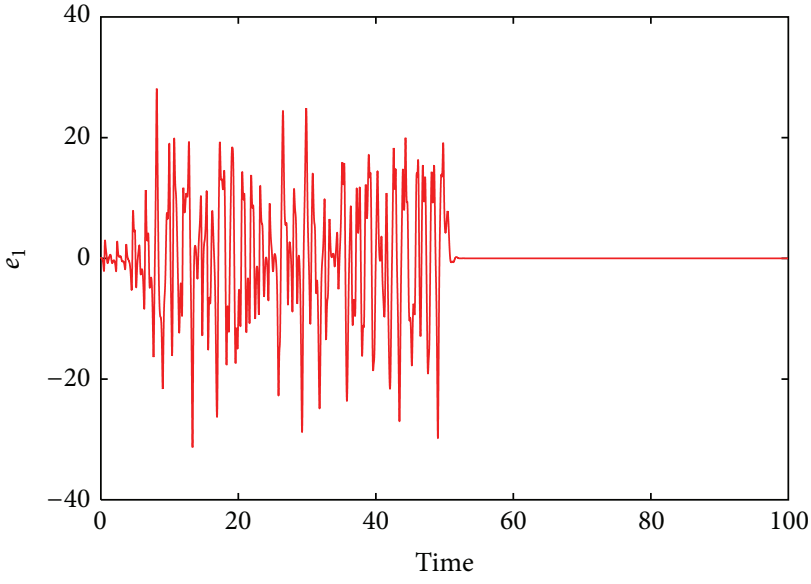

(a)

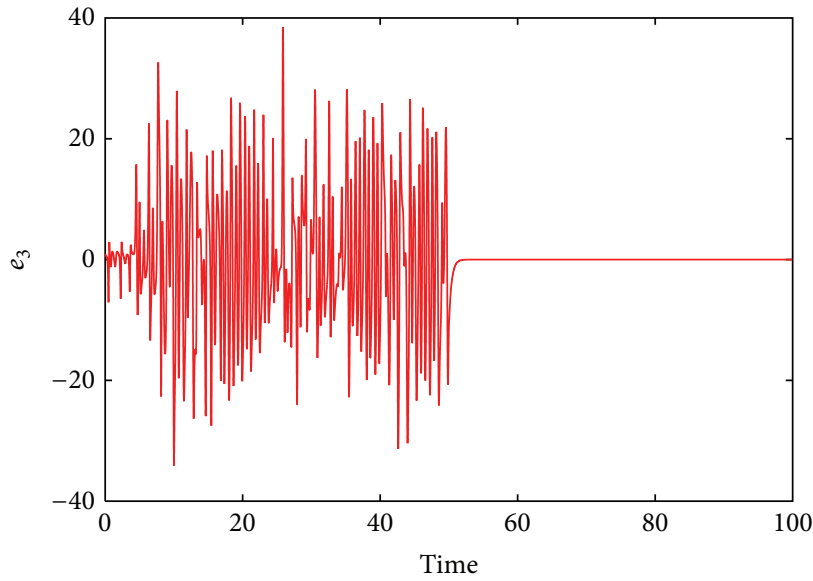

(c)

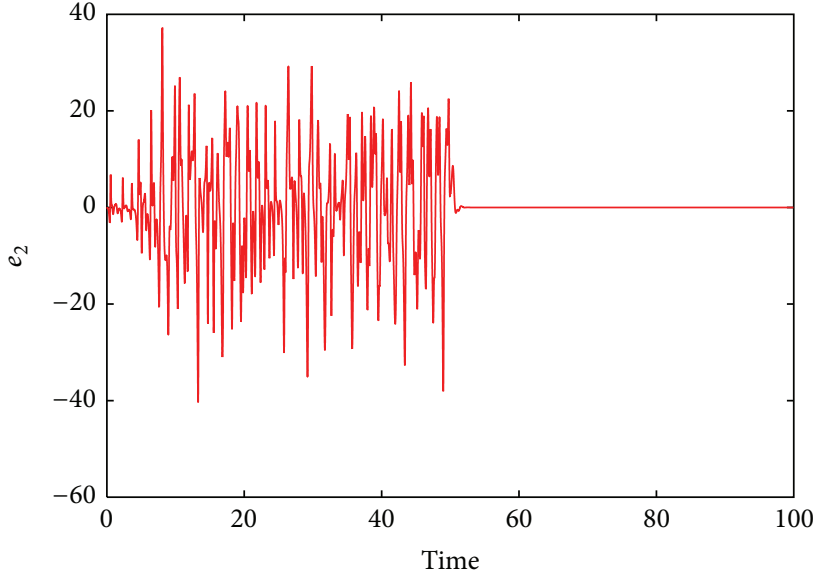

(b)

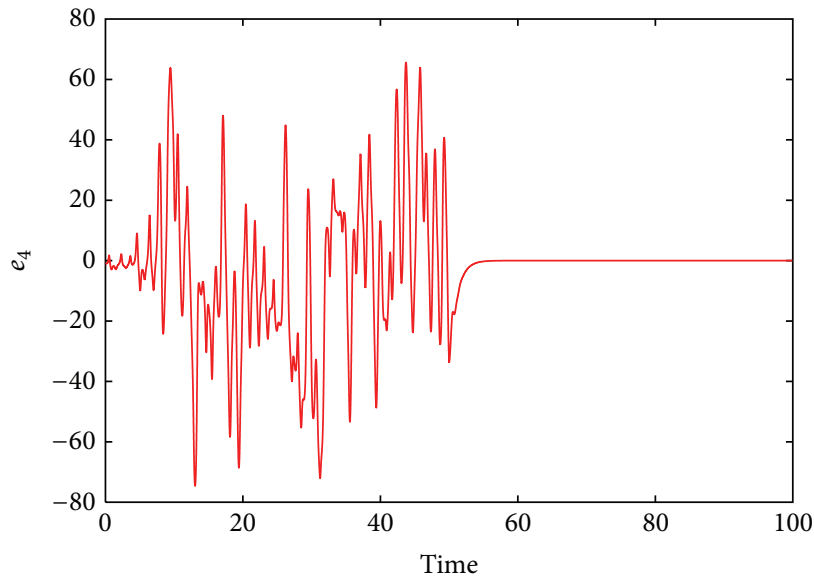

(d)

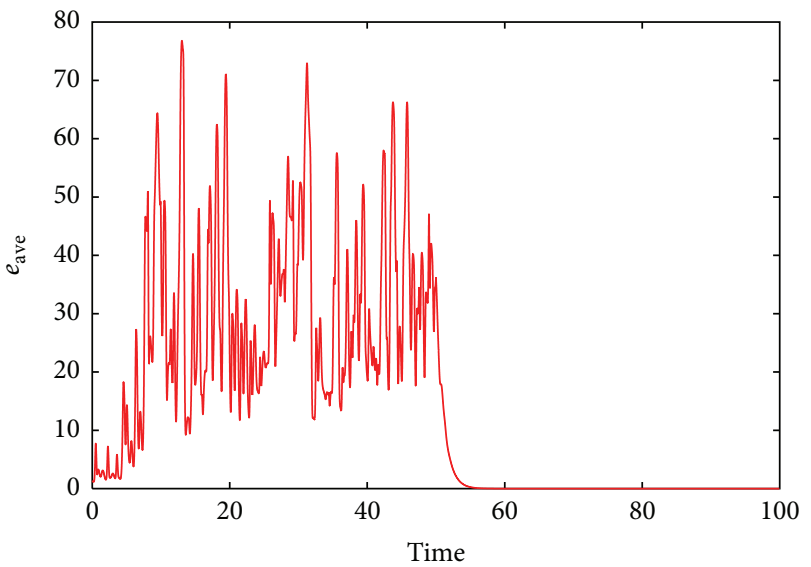

(e)

FiguRE 9: Error dynamics between the two hyperchaotic Lorenz systems with the controller deactivated for $0<t<50$ and activated for $t \geq 50$. The parameters of the system are as shown in Figure 2.

\section{Extended Backstepping Synchronization of the Lorenz Hyperchaotic System}

6.1. Design of the Extended Backstepping Controller. Letting $x=x_{1}, y=x_{2}, z=x_{3}$, and $w=x_{4}$, we recast then (2) as

$$
\begin{aligned}
& \dot{x}_{1}=a\left(x_{2}-x_{1}\right) \\
& \dot{x}_{2}=b x_{1}-x_{2}-x_{1} x_{3}+x_{4} \\
& \dot{x}_{3}=-c x_{3}+x_{1} x_{2} \\
& \dot{x}_{4}=k_{1} x_{1}+k_{2} x_{2} .
\end{aligned}
$$




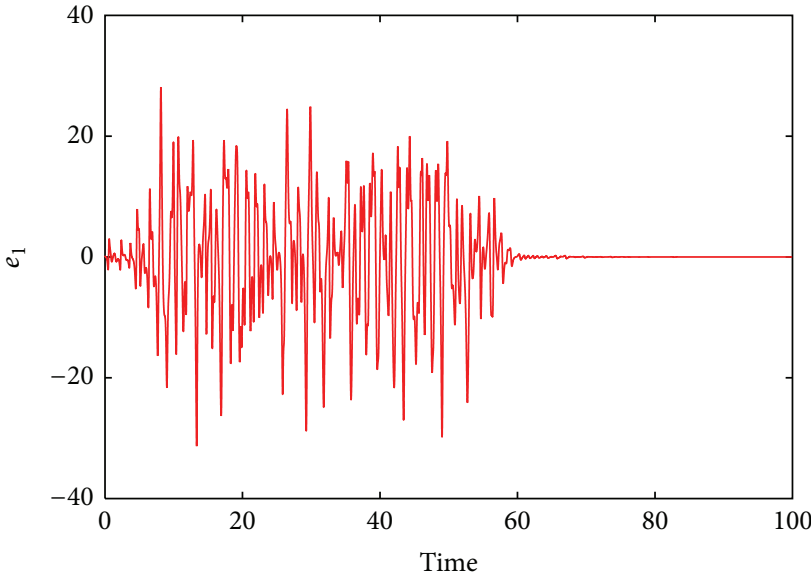

(a)

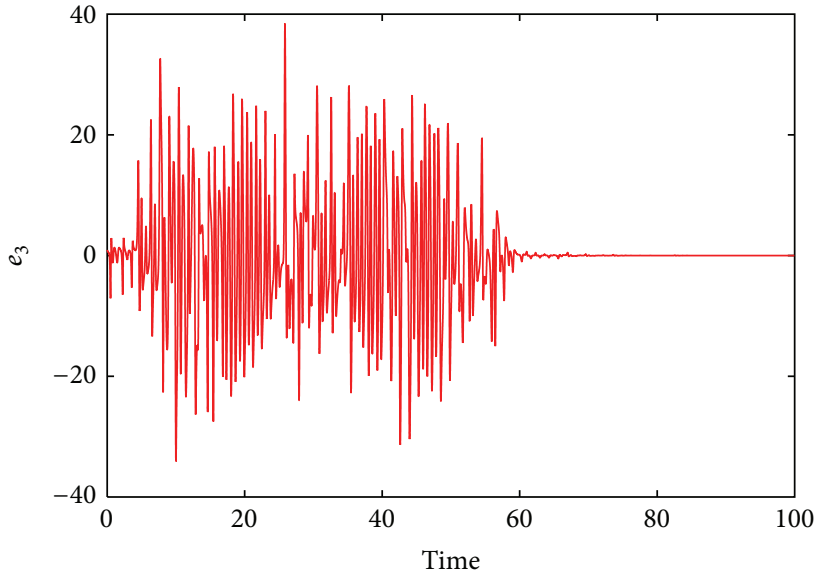

(c)

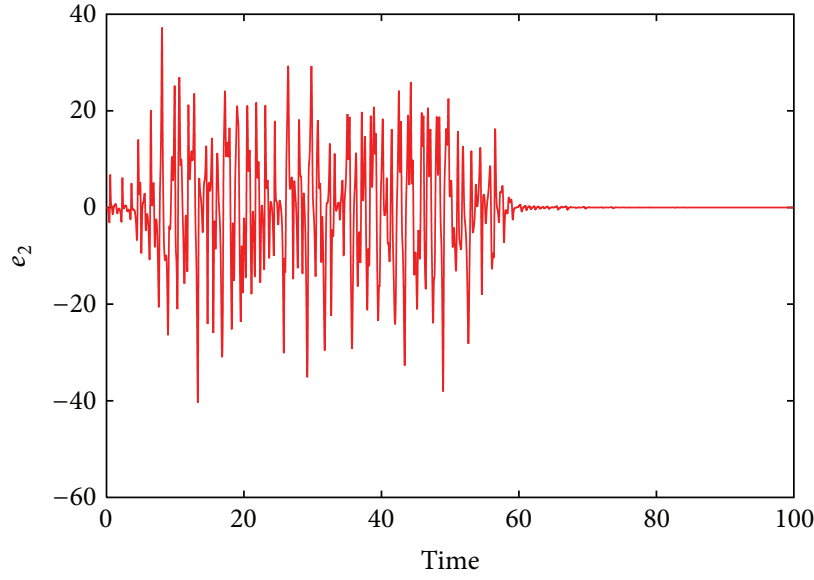

(b)

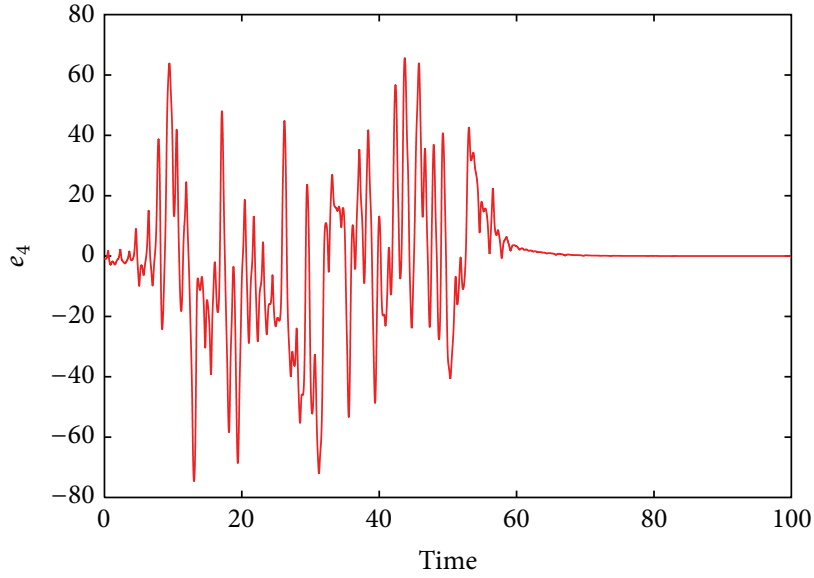

(d)

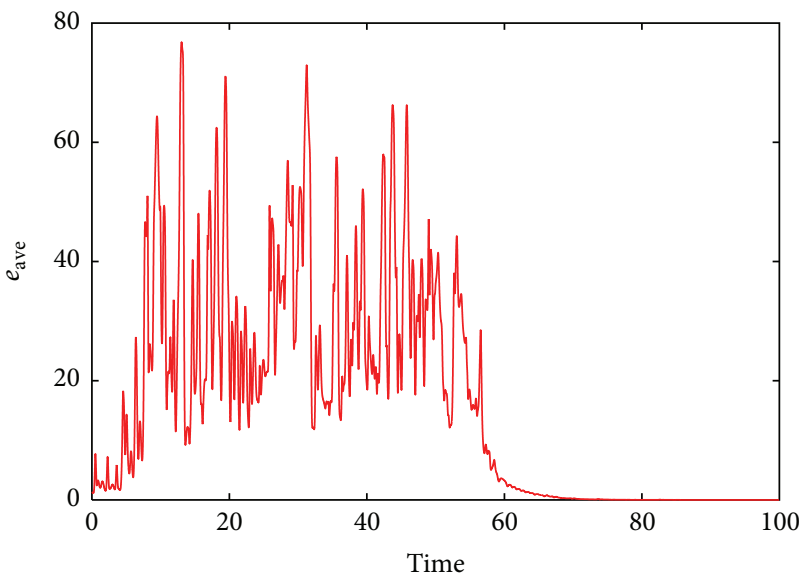

(e)

Figure 10: Error dynamics between the two hyperchaotic Lorenz systems with the controller deactivated for $0<t<50$ and activated for $t \geq 50$. The parameters of the system are as shown in Figure 2 with $\varepsilon_{i}=0.3$, where $i=1,2,3,4$.

Let system (40) be the drive system and let the following be the response system:

$$
\dot{y}_{1}=a\left(y_{2}-y_{1}\right)+u_{1}(t)
$$

$$
\begin{aligned}
& \dot{y}_{2}=b y_{1}-y_{2}-y_{1} y_{3}+y_{4}+u_{2}(t) \\
& \dot{y}_{3}=-c y_{3}+y_{1} y_{2}+u_{3}(t) \\
& \dot{y}_{4}=k_{1} y_{1}+k_{2} y_{2}+u_{4}(t),
\end{aligned}
$$


where $u_{i}(t), i=1,2,3,4$, are the control functions. Subtract (40) from (41) and use the definition of error vector as

$$
e_{i}=y_{i}-x_{i}, \quad i=1,2,3,4
$$

The error system is obtained as

$$
\begin{aligned}
& \dot{e}_{1}=a\left(e_{2}-e_{1}\right)+u_{1} \\
& \dot{e}_{2}=b e_{1}-e_{2}+e_{4}-x_{1} e_{3}-x_{3} e_{1}-e_{1} e_{3}+u_{2} \\
& \dot{e}_{3}=-c e_{3}+x_{1} e_{2}+x_{2} e_{1}+e_{1} e_{2}+u_{3} \\
& \dot{e}_{4}=k_{1} e_{1}+k_{2} e_{2}+u_{4}(t) .
\end{aligned}
$$

The objective is to find the control functions $u_{i}(t), I=$ $1,2,3,4$, that can stabilize the error states in (43) at the equilibrium point $(0,0,0,0,0)$. Firstly, we stabilize the first equation in (43) by regarding $e_{2}$ as a controller, choosing a Lyapunov function $V_{1}\left(e_{1}\right)=(1 / 2) e_{1}^{2}$, and differentiating it with respect to time to have

$$
\dot{V}_{1}=e_{1} \dot{e}_{1}=e_{1}\left[a\left(e_{2}-e_{1}\right)+u_{1}\right] \text {. }
$$

We estimate that the controller $e_{2}=\alpha_{1}\left(e_{1}\right)$; then (44) can be written as $\dot{V}_{1}=e_{1} \dot{e}_{1}=e_{1}\left[a\left(\alpha_{1}\left(e_{1}\right)-e_{1}\right)+u_{1}\right]$. If $u_{1}=0$ and the estimative function $\alpha_{1}\left(e_{1}\right)=0$, then $\dot{V}_{1}=-a e_{1}^{2}$ is negative definite ( since $a>0$ ), and hence the $e_{1}$ subsystem is stabilized. The error $\omega_{2}$ between $e_{2}$ and $\alpha_{1}\left(e_{1}\right)$ is

$$
\omega_{2}=e_{2}-\alpha_{1}\left(e_{1}\right)=e_{2}
$$

Substitution of $\dot{e}_{2}$ and $e_{2}$ from (43) and (45), respectively, into the time derivative of (45) yields

$$
\dot{\omega}_{2}=\left(b-x_{3}\right) e_{1}-\omega_{2}+e_{4}-\left(x_{1}+e_{1}\right) e_{3}+u_{2} .
$$

We now stabilize the $\left(e_{1}, \omega_{2}\right)$ subsystem given by $(46)$ as follows. We choose a Lyapunov function $V_{2}\left(e_{1}, \omega_{2}\right)=V_{1}\left(e_{1}\right)+$ $(1 / 2) \omega_{2}^{2}$ and obtain its time derivative as

$$
\dot{V}_{2}=\dot{V}_{1}\left(e_{1}\right)+\omega_{2} \dot{\omega}_{2}
$$

From (43) and (44),

$$
\dot{V}_{1}\left(e_{1}\right)=-a e_{1}^{2}+a e_{1} \dot{\omega}_{2} .
$$

Hence,

$$
\begin{aligned}
\dot{V}_{2}= & -a e_{1}^{2}+a e_{1} \dot{\omega}_{2} \\
& +\omega_{2}\left[\left(b-x_{3}\right) e_{1}-\omega_{2}+e_{4}-\left(x_{1}+e_{1}\right) e_{3}+u_{2}\right] .
\end{aligned}
$$

Estimating that the controller $e_{3}=\alpha_{2}\left(e_{1}, \omega_{2}\right)$, then (49) becomes $\dot{V}_{2}=-a e_{1}^{2}+a e_{1} \dot{\omega}_{2}+\omega_{2}\left[\left(b-x_{3}\right) e_{1}-\omega_{2}+e_{4}-\left(x_{1}+e_{1}\right) \alpha_{2}+\right.$ $u_{2}$ ]. If $u_{2}=-\left(b-x_{3}+a\right) e_{1}-e_{4}$ and the estimative function $\alpha_{2}\left(e_{1}, \omega_{2}\right)=0$, then $\dot{V}_{2}=-a e_{1}^{2}-\omega_{2}^{2}$ is negative definite (since $a, b>0)$, and hence the $\left(e_{1}, \omega_{2}\right)$ subsystem is stabilized. The error $\omega$ between $e_{3}$ and $\alpha_{2}\left(e_{1}, \omega_{2}\right)$ is

$$
\omega_{3}=e_{3}-\alpha_{2}\left(e_{1}, \omega_{2}\right)=e_{3} \text {. }
$$

Substituting $\dot{e}_{3}, e_{3}$, and $\omega_{2}$ from (43), (50), and (45), respectively, into the time derivative of (40) yields

$$
\dot{\omega}_{3}=-c \omega_{3}+\left(x_{1}+\omega_{2}\right) e_{1}+x_{1} \omega_{2}+u_{3} \text {. }
$$

We now stabilize the $\left(e_{1}, \omega_{2}, \omega_{3}\right)$ subsystem given by (50) and (51) as follows. We choose a Lyapunov function $V_{3}\left(e_{1}, \omega_{2}, \omega_{3}\right)=V_{2}\left(e_{1}, \omega_{2}\right)+(1 / 2) \omega_{3}^{2}$ and obtain its time derivative as

$$
\dot{V}_{3}\left(e_{1}, \omega_{2}, \omega_{3}\right)=\dot{V}_{2}\left(e_{1}, \omega_{2}\right)+\omega_{3} \dot{\omega}_{3} .
$$

From (51) and (52),

$$
\begin{aligned}
\dot{V}_{3}= & -a \dot{e}_{1}^{2}-\omega_{2}^{2} \\
& +\omega_{3}\left[-c \omega_{3}+\left(x_{1}+\omega_{2}\right) e_{1}+x_{1} \omega_{2}+u_{3}\right] .
\end{aligned}
$$

Choose

$$
u_{3}=-\left(x_{1}+\omega_{2}\right) e_{1}-x_{1} \omega_{2} .
$$

Then (53) reduces to $\dot{V}_{3}=-a \dot{e}_{1}^{2}-\omega_{2}^{2}-c \omega_{3}^{2}$ is negative definite (since $a, c>0)$ and hence the $\left(e_{1}, \omega_{2}, \omega_{3}\right)$ subsystem is stabilized. The error $\omega_{4}$ between $e_{4}$ and $\alpha_{3}\left(e_{1}, \omega_{2}, \omega_{3}\right)$ is

$$
\omega_{4}=e_{4}-\alpha_{3}\left(e_{1}, \omega_{2}, \omega_{3}\right) \text {. }
$$

Estimating that $\alpha_{3}\left(e_{1}, \omega_{2}, \omega_{3}\right)=-\left(k_{1} / a\right) e_{1}$, (55) becomes

$$
\omega_{4}=e_{4}+\frac{k_{1}}{a} e_{1} \text {. }
$$

Substituting $\dot{e}_{4}, \dot{e}_{1}$, and $\dot{\omega}_{2}$ from (43) and (45), respectively, into the time derivative of (56) yields

$$
\dot{\omega}_{4}=\left(k_{1}+k_{2}\right) \omega_{2}+u_{4} .
$$

We now stabilize the $\left(e_{1}, \omega_{2}, \omega_{3}, \omega_{4}\right)$ complete system given by (45), (56), and (57) as follows.

We choose a Lyapunov function $V_{4}\left(e_{1}, \omega_{2}, \omega_{3}, \omega_{4}\right)=$ $V_{3}\left(e_{1}, \omega_{2}, \omega_{3}\right)+(1 / 2) \omega_{4}^{2}$ and obtain its time derivative as

$$
\dot{V}_{4}\left(e_{1}, \omega_{2}, \omega_{3}, \omega_{4}\right)=\dot{V}_{3}\left(e_{1}, \omega_{2}, \omega_{3}\right)+\omega_{4} \dot{\omega}_{4} .
$$

Hence,

$$
\dot{V}_{4}=-a e_{1}^{2}-\omega_{2}^{2}-c \omega_{3}^{2}+\omega_{4}\left[\left(k_{1}+k_{2}\right) \omega_{2}+u_{4}\right] .
$$

Estimating that, $u_{4}=-\left(k_{1}+k_{2}\right) \omega_{2}-\omega_{4}$, then (59) becomes $\dot{V}_{4}=-a e_{1}^{2}-\omega_{2}^{2}-c \omega_{3}^{2}-\omega_{4}^{2}$ is negative definite (since $a, c>0$ ); hence systems (40) and (41) are globally synchronized. Thus, the synchronization goal is achieved with the weight $\varepsilon_{i}, i=$ $1,2,3,4$, added to the control function as follows:

$$
\begin{aligned}
& u_{1}(t)=0 \\
& u_{2}(t)=-\left(b-x_{3}+a\right) e_{1}-e_{4} \\
& u_{3}(t)=-\left(x_{2}+\omega_{2}\right) e_{1}-x_{1} \omega_{2} \\
& u_{4}(t)=-\left(k_{1}+k_{2}\right) \omega_{2}-\omega_{4} .
\end{aligned}
$$


In order to reduce controller complexity, we introduce weights to the above controllers to obtain the following:

$$
\begin{aligned}
& u_{1}(t)=[0] \varepsilon_{1} \\
& u_{2}(t)=\left[-\left(b-x_{3}+a\right) e_{1}-e_{4}\right] \varepsilon_{2} \\
& u_{3}(t)=\left[-\left(x_{2}+\omega_{2}\right) e_{1}-x_{1} \omega_{2}\right] \varepsilon_{3} \\
& u_{4}(t)=\left[-\left(k_{1}+k_{2}\right) \omega_{2}-\omega_{4}\right] \varepsilon_{4} .
\end{aligned}
$$

6.2. Numerical Simulation. Using the fourth-order RungeKutta algorithm with initial conditions $\left(x_{1}, x_{2}, x_{3}, x_{4}\right)=$ $(0.8,-0.8,-1.0,1.0) ;\left(y_{1}, y_{2}, y_{3}, y_{4}\right)=(1.0,-1.0,-0.1,0.1)$, a time step of 0.001 , and fixing the parameter values as in Figure 2 to ensure hyperchaotic dynamics of the state variables, we solved systems (40) and (41) with the control function as defined in (60) and (61). The result obtained shows that the error state variable moves hyperchaotically with time when the controller is switched off and when the controller is activated at $t=50$ (see Figures 9 and 10), the error state variables converge to zero, thereby guaranteeing the synchronization of systems (40) and (41). This is confirmed by the synchronization quality $e$ given by

$$
e=\sqrt{e_{1}^{2}+e_{2}^{2}+e_{3}^{2}+e_{4}^{2}}
$$

In Figure 10, we set the weights, $\varepsilon_{i}=0.3$, and found that one needs only about $70 \%$ of the energy required by the controllers in (60). Hence, hyperchaotic system, studied in this work, achieves stable synchronization faster than when weights are introduced to the usual backstepping controllers. The proposed scheme could be very useful and it can be suitable for practical implementations in other hyperchaotic systems with broadband spectrum.

\section{Conclusion}

We have applied the extended backstepping techniques to control and track any smooth function, $f(t)$, and to synchronize chaotic and hyperchaotic Lorenz systems. The results obtained showed that all the generalized recursive backstepping controllers with the extended scheme were effective in the stabilization and tracking control of the chaotic Lorenz system with weight $\varepsilon \geq 0.5$ and hyperchaotic Lorenz system with $\varepsilon \geq 0.7$. The extended backstepping controllers achieved synchronization between two identical chaotic Lorenz systems with $\varepsilon \geq 0.3$ and two identical hyperchaotic Lorenz systems with $\varepsilon \geq 0.3$. This implies that the proposed extended backstepping techniques reduced control strength in the designed controllers between $30 \%$ and $70 \%$, thereby producing economic controllers with low energy consumption and reduced complexity, which are suitable for practical implementation. In various branches of science and technology, efforts are geared towards reducing energy consumption; thus, practical implementation of the proposed scheme shall be very useful. Future work shall be focused on practical implementation of the proposed scheme.

\section{Conflict of Interests}

The authors declare that there is no conflict of interests regarding the publication of this paper.

\section{References}

[1] C. Kyrtsou and W. C. Labys, "Evidence for chaotic dependence between US inflation and commodity prices," Journal of Macroeconomics, vol. 28, no. 1, pp. 256-266, 2006.

[2] C. Kyrtsou and W. C. Labys, "Detecting positive feedback in multivariate time series: the case of metal prices and US inflation," Physica A: Statistical Mechanics and its Applications, vol. 377, no. 1, pp. 227-229, 2007.

[3] S. H. Kellert, In the Wake of Chaos: Unpredictable Order in Dynamical Systems, University of Chicago Press, 1993.

[4] E. S. James, M. Mark, V. Tomas, and M. Mirna, Application of Chaos Theory to Biology and Medicine, vol. 27, Baylor College of Medicine, Houston, Tex, USA, 1999.

[5] M. Berry, Quantum Chaology, Weiderfeld and Nicolson, Bristol, UK, A guide for the perplexed by Jim Al-khalili, 2008.

[6] E. M. Adilson, "General relativity and quantum cosmology: astrophysics," Physical Review Letters, vol. 91, Article ID 231101, 2003.

[7] A. N. Njah, "Tracking control and synchronization of the new hyperchaotic Liu system via backstepping techniques," Nonlinear Dynamics, vol. 61, no. 1-2, pp. 1-9, 2010.

[8] E. Scholl and H. G. Schuster, Handbook of Chaos Control, WileyVCH, 2nd edition, 2007.

[9] L. M. Pecora and T. L. Carroll, "Synchronization in chaotic systems," Physical Review Letters, vol. 64, no. 8, pp. 821-824, 1990.

[10] M. Zhan, X. Wang, X. Gong, G. W. Wei, and C.-H. Lai, "Complete synchronization and generalized synchronization of one-way coupled time-delay systems," Physical Review E: Statistical, Nonlinear, and Soft Matter Physics, vol. 68, no. 3, Article ID 036208, 2003.

[11] O. I. Olusola, U. E. Vincent, and A. N. Njah, "Synchronization, multistability and basin crisis in coupled pendula," Journal of Sound and Vibration, vol. 329, no. 4, pp. 443-456, 2010.

[12] L. Dianchen, W. Aicheng, and T. Xiandon, "Control and synchronization of a new hyperchaotic system with unknown parameters," International Journal of Nonlinear Science, vol. 6, no. 3, pp. 224-229, 2008.

[13] U. E. Vincent, "Chaos synchronization using active control and backstepping control: a comparative analysis," Non-Linear Analysis: Theory, Method and Applications, vol. 13, pp. 256-261, 2008.

[14] E. W. Bai and K. E. Longngren, "Synchronization of two Lorenz systems using active control," Chaos Solitions and Fractals, vol. 8, no. 1, pp. 51-58, 1997.

[15] C. Li, X. Liao, and K.-W. Wong, "Lag synchronization of hyperchaos with application to secure communications," Chaos, Solitons and Fractals, vol. 23, no. 1, pp. 183-193, 2005.

[16] X. Wang and Y. Wang, "Adaptive control for synchronization of a four-dimensional chaotic system via a single variable," Nonlinear Dynamics, vol. 65, no. 3, pp. 311-316, 2011.

[17] S. Zhigong, Z. Wenzhi, and S. Gangquan, "Adaptive synchronization design for uncertain systems in the presence of unknown parameters," Nonlinear Dynamics, vol. 72, pp. 729749, 2013. 
[18] O. I. Olusola, U. E. Vincent, A. N. Njah, and E. Ali, "Control and synchronization of chaos in Biological systems via backstepping design," International Journal of Nonlinear Science, vol. 11, pp. 121-128, 2011.

[19] S. Mascolo, "Backstepping design for controlling Lorenz chaos," in Proceedings of the 36th IEEE Conference on Decision and Control, pp. 1500-1501, San Diego, Calif, USA, December 1997. 

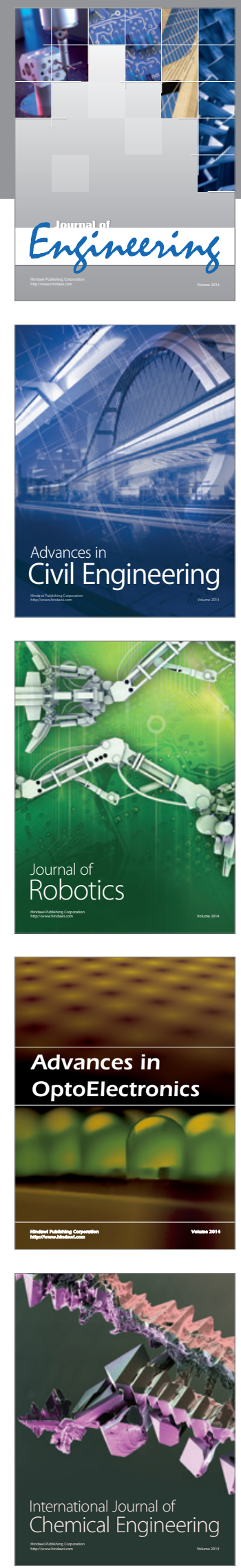

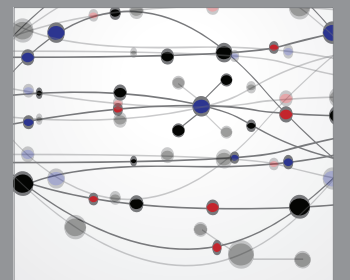

The Scientific World Journal
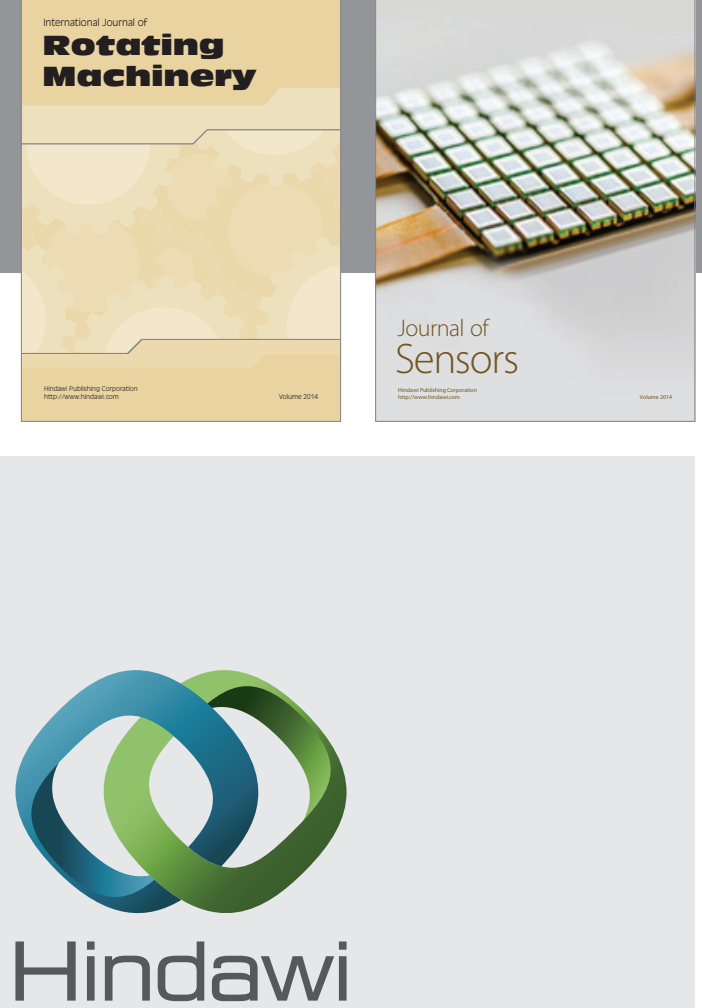

Submit your manuscripts at http://www.hindawi.com
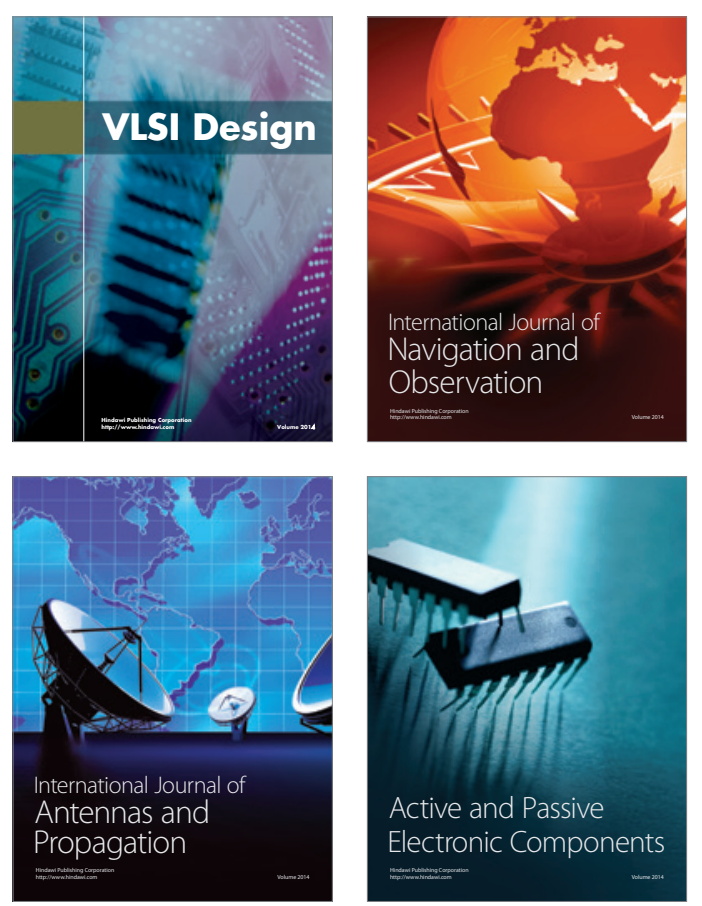
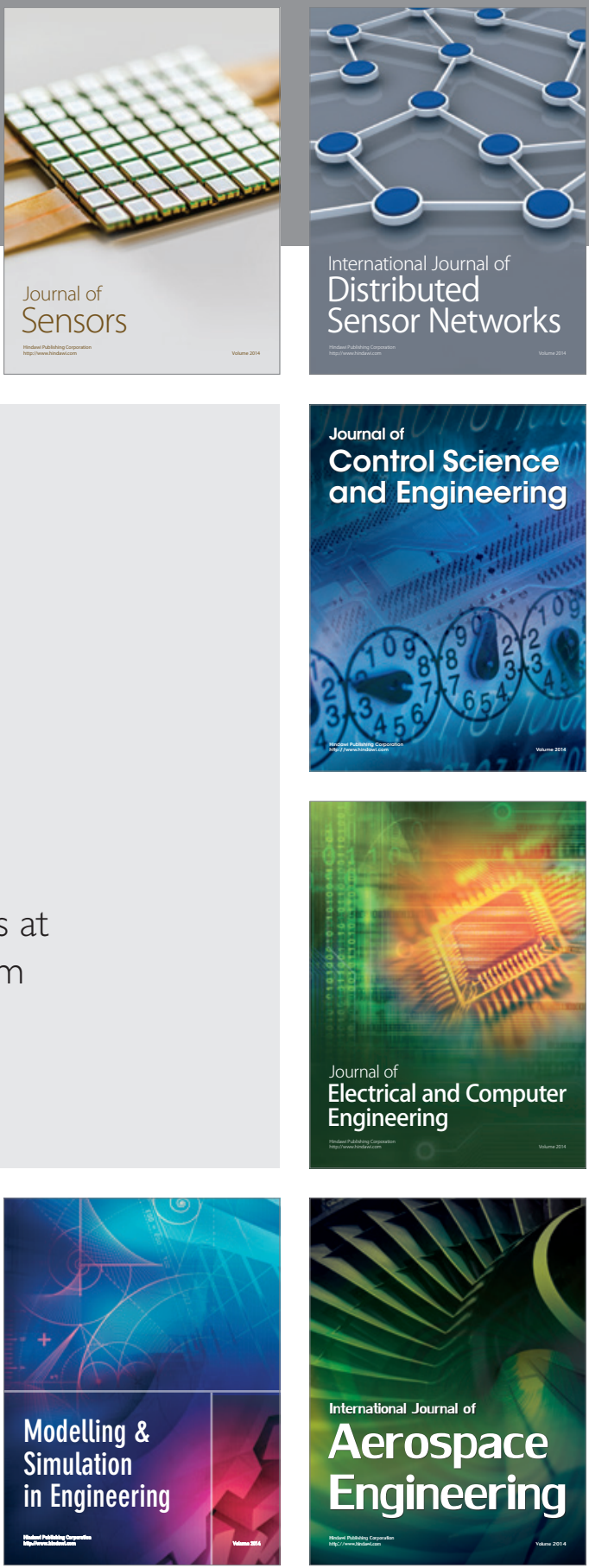

Journal of

Control Science

and Engineering
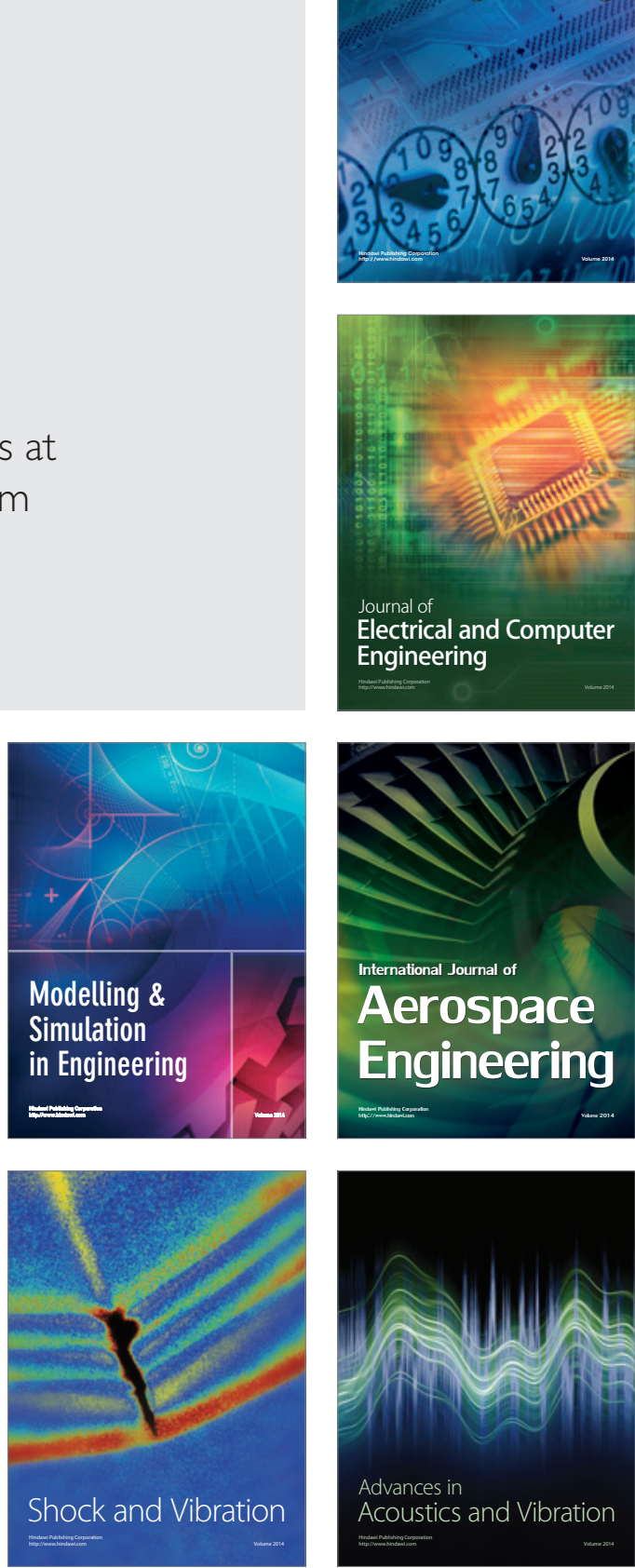